\title{
UNIVERSITYOF
}

FORWARD

THINKING

WESTMINSTER用

WestminsterResearch

http://www.westminster.ac.uk/westminsterresearch

Study on the pulmonary delivery system of apigenin loaded albumin nanocarriers with antioxidant activity

Papay, Z.E., Kosa, A., Boddi, B., Merchant, Z., Saleem, I.Y., Zariwala, M., Klebovich, I., Somavarapu, S. and Antal, I.

This is an author's accepted manuscript of an article published in the Journal of Aerosol Medicine and Pulmonary Drug Delivery doi:10.1089/jamp.2016.1316.

The final definitive version is available online at:

https://dx.doi.org/10.1089/jamp.2016.1316

The WestminsterResearch online digital archive at the University of Westminster aims to make the research output of the University available to a wider audience. Copyright and Moral Rights remain with the authors and/or copyright owners.

Whilst further distribution of specific materials from within this archive is forbidden, you may freely distribute the URL of WestminsterResearch: ((http://westminsterresearch.wmin.ac.uk/).

In case of abuse or copyright appearing without permission e-mail repository@westminster.ac.uk 


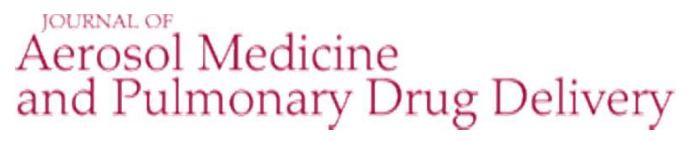

Journal of Aerosol Medicine and Pulmonary Drug Delivery; http://mc.manuscriptcentral.com/aerosol

\section{Study on the pulmonary delivery system of apigenin loaded albumin nanocarriers with antioxidant activity}

\begin{tabular}{|c|c|}
\hline Journal: & Journal of Aerosol Medicine and Pulmonary Drug Delivery \\
\hline Manuscript ID & JAMP-2016-1316.R2 \\
\hline Manuscript Type: & Original Research \\
\hline Date Submitted by the Author: & 03-Jan-2017 \\
\hline Complete List of Authors: & $\begin{array}{l}\text { Papay, Zsofia; Semmelweis University, Department of Pharmaceutics } \\
\text { Kosa, Annamaria; Institute of Biology, Eötvös Lóránd University, } \\
\text { Department of Plant Anatomy } \\
\text { Boddi, Bela; Institute of Biology, Eötvös Lóránd University, Department of } \\
\text { Plant Anatomy } \\
\text { Merchant, Zahra; University College London School of Pharmacy } \\
\text { Saleem, Imran; Liverpool John Moores University, School of Pharmacy \& } \\
\text { Biomolecular Sciences } \\
\text { Zariwala, Mohammed ; University of Westminster } \\
\text { Klebovich, Imre; Semmelweis University, Department of Pharmaceutics } \\
\text { Somavarapu, Satyanarayana ; University College London School of } \\
\text { Pharmacy } \\
\text { Antal, Istvan; Semmelweis Egyetem, Department of Pharmaceutics }\end{array}$ \\
\hline Keyword: & Aerosol Distribution, inhaled therapy, Modeling \\
\hline $\begin{array}{r}\text { Manuscript Keywords (Search } \\
\text { Terms): }\end{array}$ & flavonoid, apigenin, albumin nanoparticles \\
\hline
\end{tabular}

\section{SCHOLARONE ${ }^{\text {m }}$ \\ Manuscripts}




\section{Study on the pulmonary delivery system of apigenin loaded albumin nanocarriers with antioxidant activity}

Zsófia Edit Pápay ${ }^{1}$, Annamária Kósa, $\mathrm{PhD}^{2}$, Béla Böddi, $\mathrm{PhD}^{2}$, Zahra Merchant ${ }^{3}$, Imran Y Saleem, $\mathrm{PhD}{ }^{4}$, Mohammed Gulrez Zariwala, $\mathrm{PhD}^{5}$, Imre Klebovich, $\mathrm{PhD}{ }^{1}$, Satyanarayana Somavarapu, $\mathrm{PhD}^{3 *}$, István Antal, $\mathrm{PhD}^{1^{*}}$

${ }^{1}$ Department of Pharmaceutics, Semmelweis University, Hőgyes E. Street 7-9, H-1092 Budapest, Hungary

2 Department of Plant Anatomy, Institute of Biology, Eötvös Lóránd University, Pázmány Péter Street 1/C, Budapest, Hungary

${ }^{3}$ Department of Pharmaceutics, UCL School of Pharmacy, 29-39 Brunswick Square, London WC1N 1AX, United Kingdom

${ }^{4}$ Formulation and Drug Delivery Research, School of Pharmacy and Biomolecular Sciences, Liverpool John Moores University, Liverpool, United Kingdom

5 Department of Biomedical Science, Faculty of Science and Technology, University of Westminster, 115 New Cavendish Street, London, W1W 6UW, United Kingdom

* To whom correspondence should be addressed:

antal.istvan@pharma.semmelweis-univ.hu

Department of Pharmaceutics

Semmelweis University

Högyes E. Street 7-9,

H-1092 Budapest, Hungary

Tel/Fax: +3612017-0914; Tel.: +361476-3600/53066, 53087

Running title:

Albumin-Apigenin Nanoparticles against Lung Injury 
1 Study on the pulmonary delivery system of apigenin loaded albumin nanocarriers with antioxidant activity

3 Zsófia Edit Pápay ${ }^{1}$, Annamária Kósa, $\mathrm{PhD}^{2}$, Béla Böddi, $\mathrm{PhD}^{2}$, Zahra Merchant ${ }^{3}$, Imran Y

4 Saleem, $\mathrm{PhD}^{4}$, Mohammed Gulrez Zariwala, $\mathrm{PhD}^{5}$, Imre Klebovich, $\mathrm{PhD}{ }^{1}$, Satyanarayana

5 Somavarapu, $\mathrm{PhD}^{3 *}$, István Antal, $\mathrm{PhD}^{1^{*}}$

$6{ }^{1}$ Department of Pharmaceutics, Semmelweis University, Hőgyes E. Street 7-9, H-1092

7 Budapest, Hungary

$8{ }^{2}$ Department of Plant Anatomy, Institute of Biology, Eötvös Lóránd University, Pázmány

9 Péter Street 1/C, Budapest, Hungary

$10{ }^{3}$ Department of Pharmaceutics, UCL School of Pharmacy, 29-39 Brunswick Square, London 11 WC1N 1AX, United Kingdom

$12{ }^{4}$ Formulation and Drug Delivery Research, School of Pharmacy and Biomolecular Sciences, 13 Liverpool John Moores University, Liverpool, United Kingdom

$14{ }^{5}$ Department of Biomedical Science, Faculty of Science and Technology, University of 15 Westminster, 115 New Cavendish Street, London, W1W 6UW, United Kingdom

$16 *$ To whom correspondence should be addressed:

17 antal.istvan@pharma.semmelweis-univ.hu

18 Department of Pharmaceutics

19 Semmelweis University

20 Hőgyes E. Street 7-9,

21 H-1092 Budapest, Hungary

22 Tel/Fax: +3612017-0914; Tel.: +361476-3600/53066, 53087

23 Running title:

24 Albumin-Apigenin Nanoparticles against Lung Injury 25 
Background: Respiratory diseases are mainly derived from acute and chronic inflammation of the alveoli and bronchi. The pathophysiological mechanisms of pulmonary inflammation

\section{ABSTRACT}

mainly arise from oxidative damage that could ultimately lead to acute lung injury (ALI). Apigenin (Api) is a natural polyphenol with prominent antioxidant and anti-inflammatory properties in the lung. Inhalable formulations consist of nanoparticles (NPs) have several advantages over other administration routes therefore this study investigated the application of apigenin loaded bovine serum albumin nanoparticles (BSA-Api-NPs) for pulmonary delivery.

Methods: Dry powder formulations of BSA-Api-NPs were prepared by spray drying and characterized by laser diffraction particle sizing, scanning electron microscopy, differential scanning calorimetry and powder X-ray diffraction. The influence of dispersibility enhancers (lactose monohydrate and L-leucine) on the in vitro aerosol deposition using a next generation impactor (NGI) was investigated in comparison to excipient-free formulation. The dissolution of Api was determined in simulated lung fluid by using Franz cell apparatus. The antioxidant activity was determined by 2,2-Diphenyl-1-picrylhydrazyl $\left(\mathrm{DPPH}^{\bullet}\right)$ free radical scavenging assay.

Results: The encapsulation efficiency and the drug loading was measured to be $82.61 \pm$ $4.56 \%$ and $7.51 \pm 0.415 \%$. The optimized spray drying conditions were suitable to produce particles with low residual moisture content. The spray dried BSA-Api-NPs possessed good the aerodynamic properties due to small and wrinkled particles with low mass median aerodynamic diameter, high emitted dose and fine particle fraction. The aerodynamic properties was enhanced by leucine and decreased by lactose, however, the dissolution was reversely affected. The $\mathrm{DPPH}^{*}$ assay confirmed that the antioxidant activity of encapsulated Api was preserved. 
49 Conclusion: This study provides evidence to support that albumin nanoparticles are suitable

50 carriers of Api and the use of traditional or novel excipients should be taken into

51 consideration. The developed BSA-Api-NPs is a novel delivery system against lung injury

52 with potential antioxidant activity.

53 Keywords: aerosol distribution, inhaled therapy, modeling

54 


\section{INTRODUCTION}

Respiratory diseases are thought to be mainly derived from acute and chronic inflammation of the alveoli and the bronchi. The pathophysiological mechanisms of pulmonary inflammation arise from several factors, including oxidative damage due to cytotoxic mediators that may ultimately lead to acute lung injury (ALI), acute respiratory distress syndrome (ARDS) and cancer ${ }^{1}$. A growing body of scientific data suggests that natural occurring compounds possess preventive and therapeutic properties with inherent low toxicity $^{2}$. Among phytochemicals, apigenin (Api, Figure 1) is a promising candidate as a therapeutic agent, mainly due to its antioxidant and anti-inflammatory properties ${ }^{3-6}$. It has been demonstrated that Api has protective effects against bleomycin-induced lung fibrosis in rats which is associated with its antioxidant and anti-inflammatory capacities ${ }^{7}$. Another study provided evidence that Api has was able to decrease oxidative stress and inflammation on paraquat-induced ALI in mice ${ }^{8}$ and reduced the pathological alterations of pulmonary tissue in acute pancreatitis associated ALI, therefore suggesting protection in the lung 9 . Furthermore, Api has anti-inflammatory effect owing to significant inhibition of proinflammatory cytokines, activator protein (AP-1) and cyclooxygenase-2 (COX-2) in human pulmonary epithelial cells ${ }^{10}$ and in mice as well ${ }^{11}$. However, Api's has low water solubility $(2.16 \mu \mathrm{g} / \mathrm{ml}$ at $\mathrm{pH}$ 7.5) and therefore it was recently classified as BCS (Biopharmaceutical Classification System) II. drug ${ }^{12}$.

Encapsulation and delivery of phytoconstituents with health effects has attracted much attention in recent years. Developing a suitable carrier system is essential to improve the overall activity and reduce the possible toxicity of these agents ${ }^{13}$. Among the potential carrier systems, serum albumin nanoparticles have notable advantages including biodegradability, non-antigenicity and cell-targeting ability ${ }^{14,15}$. Moreover, albumin provide exceptional ligand binding capacity for various drugs owing to three homologous domains with two separate 
80 helical subdomains ${ }^{16}$. Studies reported the successful incorporation of flavonoids into albumin 81 nanoparticles that can improve their stability ${ }^{17}$ and antitumor activity ${ }^{18}$.

82 Pulmonary delivery of pharmacologically active ingredients are extensively studied due to prominent advantages over other delivery routes of administration ${ }^{19}$. The lungs have a large

84 surface area, limited enzymatic activity and high permeability therefore drugs can be delivered either locally for the treatment of respiratory diseases or systematically in order to e.g. avoid first pass metabolism ${ }^{20}$. Dry Powder Inhaler (DPI) products offer precise and reproducible delivery of fine drug particle fraction to the deep lung and recent studies have proved that these are more cost effective than other products ${ }^{21}$. This non-invasive delivery route could be suitable for poorly water soluble drugs in nanoparticles with increased solubility $^{22}$. It is also well recognized that nanoparticles have benefits over other carriers in the micron scale such as controlled drug release, avoiding mucociliary clearance and improve deposition $^{23,24}$. Albumin is naturally present in the body, as well as in the lung epithelium ${ }^{24}$, moreover, the body can absorb proteins into the bloodstream by transcytosis which occurs deep in the lung and allows drug molecules to pass through cell membrane ${ }^{25}$. Therefore the presence of BSA in the nanoparticle system increases membrane permeability, may facilitate epithelial cell uptake and translocation through the alveo-capillary barrier of the lung ${ }^{26}$. It was proved that albumin nanoparticles have high biocompatibility in a wide dose range and remained longer in the lungs with low systemic exposure ${ }^{24}$. Thus encapsulation of apigenin into albumin nanoparticles would enhance its solubility and distribution in the lung. However, the formulation of dry powders with optimal aerodynamic properties for pulmonary drug delivery is challenging. Spray drying is a technique for manufacturing respirable dry powders in one step. During the process, the liquid phase is atomized into droplets that dry rapidly in the drying chamber due to the compressed air. The process conditions like heat, flow rate, aspiration rate and pump rate also determine the quality of the product. The thermal 
105 degradation caused by overheating can be avoided by the rapid evaporation of the solvent ${ }^{27}$.

106 Hence, it is suitable for drying colloidal systems resulting in uniform particle morphology.

107 Nanoparticle delivery systems targeted to the lungs offer several advantages such as sustained 108 release, increased local drug concentration and targeted site of action ${ }^{28}$. Moreover, improved 109 drug solubility, uniform dose distribution and fewer side effects can be achieved, compared to 110 conventional dry powders. In general, respirable nanoparticles are embedded in microparticles 111 in aerodynamic size range ${ }^{26}$.

112 The aim of this work was to develop a novel dry powder formulation against ALI 113 caused by oxidative stress. The prepared albumin nanoparticles were characterized in terms of 114 size, zeta potential and drug loading, additionally the fluorescence properties were 115 investigated. Following this, the nanoparticles were spray dried with two types of excipients, 116 namely a traditional lactose monohydrate and a novel amino acid, L-leucine. In vitro aerosol 117 deposition patterns were determined in comparison to excipient-free formulation using a next 118 generation impactor (NGI) and dissolution test was performed in simulated lung fluid by 119 using Franz cell apparatus. Laser diffraction particle sizing, morphology and residual moisture 120 content were measured along with the antioxidant activity.

\section{MATERIALS AND METHODS}

\section{MATERIALS}

Apigenin (Api) was purchased from (purity > 99\%) Hangzhou Dadyangchem Co., Ltd. (China). Bovine serum albumin powder (BSA, purity $\geq 98 \%$ ), L-leucine, analytical grade chloroform, acetonitrile and trifluoroacetic acid (TFA) were obtained from Sigma Aldrich Ltd. (Dorset, UK). Lactohale ${ }^{\circledR}$ LH 230 was supplied by Friesland Foods Domo (Amersfoort, The Netherlands). 2,2-Diphenyl-1-picrylhydrazyl (DPPH*) free radical was purchased from Sigma-Aldrich (Darmstadt, Germany). For the solubility and drug release study, PBS buffer was purchased (Sigma Aldrich Ltd., Dorset, UK) and simulated lung fluid modified with 
$130 \quad 0.02 \%(\mathrm{w} / \mathrm{v})(\mathrm{mSLF})$ was prepared. All of the materials for the mSLF were purchased from

131 Sigma Aldrich Ltd. (Dorset, UK).

\section{METHODS}

133 2.1. Preparation of BSA-NPs

BSA nanoparticles were prepared using a nanoparticle albumin bound technology with minor modifications ${ }^{29}$. Briefly, $1000 \mathrm{mg}$ of BSA was dissolved in $50 \mathrm{ml}$ of distilled water 136 saturated with chloroform. Separately, $100 \mathrm{mg}$ of Api was dissolved in $3 \mathrm{ml}$ of chloroform 137 saturated with water and ultrasonicated for 10 minutes. These two solutions were mixed and 138 ultrasonicated for 20 minutes with a probe-type sonicator (MSE Soniprep 150 Ultrasonic 139 Processor, MSE Ltd., London, UK) on ice. After homogenization, the chloroform was 140 evaporated by rotary evaporator (Rotavapor ${ }^{\circledR}$ R-10, BÜCHI Labortechnik AG, Flawil, 141 Switzerland) at $25^{\circ} \mathrm{C}$ for 15 minutes. The obtained nanoparticles were filtered through filter 142 paper $(0.45 \mu \mathrm{m}$, Ficher Scientific Ltd., Loughborough, UK) and further spray dried.

\section{2.2. Characterization of BSA-Api-NPs}

\section{$144 \quad$ 2.2.1. Particle size and zeta potential analysis}

The average particle size and polydispersity index (PDI) were determined by dynamic

146 light scattering (DLS) using Zetasizer Nano ZS instrument (Malvern Instruments Ltd., 147 Worcestershire, UK). Zeta potential of the particles was quantified with laser doppler 148 velocimetry (LDV) using the same instrument. All measurements were performed in triplicate $149(\mathrm{n}=3)$ at $25^{\circ} \mathrm{C}$ and presented as mean \pm standard deviation (SD).

\subsubsection{Determination of drug loading and encapsulation efficiency}

To determine the amount of Api, $1 \mathrm{ml}$ sample from the BSA-Api formulation was

152 withdrawn and the apigenin content was determined in $\mathrm{mg} / \mathrm{ml}$ by adding $5 \mathrm{ml}$ of dimethyl 153 sulfoxide and methanol (DMSO:MeOH, 50:50\% v/v) and sonicated for 10 minutes. The exact 154 concentrations were determined after filtration $(0.22 \mu \mathrm{m}$ ) by HPLC 1260 (Agilent 
155 Technologies Inc., Santa Clara, USA) using reverse-phase $\mathrm{C}_{18}$ column (Phenomenex ${ }^{\circledR}$, $156250 \times 4.6 \mathrm{~mm}, 4 \mu \mathrm{m})$ as the stationary phase. The temperature was set to $25^{\circ} \mathrm{C}$. The mobile 157 phase consisted of $40 \%$ acetonitrile and $60 \%$ water containing $0.1 \%(\mathrm{v} / \mathrm{v})$ TFA. The system 158 was run isocratically at the flow rate of $1.2 \mathrm{ml} / \mathrm{min}$ and the Api was detected at $340 \mathrm{~nm}\left(\mathrm{t}_{\mathrm{R}}=\right.$ 159 8.3). The injection volume was set to $10 \mu$ l. A calibration curve was conducted by diluting 160 stock solution $(0.1 \mathrm{mg} / \mathrm{ml})$ with $\mathrm{R}^{2}$ value of 0.999 .

161 The drug loading efficiency (DL, \%) and encapsulation efficiency (EE, \%) were 162 calculated according to the equations (Eq. 1. and 2.), comparing the encapsulated Api content $163\left(\mathrm{mg} / \mathrm{ml}, \mathrm{W}_{\text {encapsulated }}\right)$ to total nanoparticle system which means the weighted amount of BSA 164 and Api together $\left(\mathrm{mg} / \mathrm{ml}, \mathrm{W}_{\text {total }}\right)$ and the amount of Api $\left(\mathrm{mg} / \mathrm{ml}, \mathrm{W}_{\text {theoretical }}\right)$ used in the 165 formulations.

\subsubsection{Fluorescence spectroscopy}

$$
\begin{gathered}
D L(\%)=\frac{W_{\text {encapsulated }}}{W_{\text {total }}} \times 100 \\
E E(\%)=\frac{W_{\text {encapsulated }}}{W_{\text {theoretical }}} \times 100
\end{gathered}
$$

The fluorescence emission spectra of BSA and BSA-Api-NPs were measured with Jobin Yvon-Horiba Fluoromax-3 (Paris, France) spectrofluorometer. The samples which contained the nanoparticles were diluted 10 times and the fluorescence emission spectra were recorded between 300 and $450 \mathrm{~nm}$ at $25{ }^{\circ} \mathrm{C}$ where the excitation wavelength was set to 285 $\mathrm{nm}$. The data collection frequency was $0.5 \mathrm{~nm}$ and the integration time was $0.2 \mathrm{~s}$. The excitation slit was set at a bandpass width of $2 \mathrm{~nm}$ and the emission slit at $5 \mathrm{~nm}$. Each spectrum was recorded three times and the mean values were calculated automatically. The SPSERV V3.14 software (C) Csaba Bagyinka, Institute of Biophysics, Biological Research Center of the Hungarian Academy of Sciences, Szeged, Hungary) was used for baseline correction, for five point linear smoothing and for the correction to the wavelength-dependent 
179 sensitivity changes of the spectrofluorometer. The subtraction of the Raman band at $390 \mathrm{~nm}$ 180 was performed.

181 To obtain the three-dimensional projections and contour maps of fluorescence spectra 182 of the samples, the fluorescence emission were recorded from 265 to $450 \mathrm{~nm}$ using different 183 excitation wavelengths from 250 to $310 \mathrm{~nm}$ with $10 \mathrm{~nm}$-steps with the same instrument 184 mentioned above. All emission scan ranges were set to start at least $15 \mathrm{~nm}$ away from the 185 corresponding excitation wavelengths. Other settings were similar as described above. Each 186 spectrum was recorded three times and the mean values were calculated automatically. The 187 three-dimensional fluorescence spectra were visualized with the software SURFER Version 18810 (Golden Software, Inc., Colorado, USA). Spectra were combined together into a three 189 dimensional surface data set with axes of excitation and emission wavelengths and 190 fluorescence intensity. Data were also converted into two dimensional contour maps.

\section{2.3. Spray drying of BSA-Api-NPs}

192 Spray drying of the BSA-Api formulations without excipient and in the presence of 193 lactose monohydrate $(50 \%, \mathrm{w} / \mathrm{w})$ and L-leucine $(9 \%, \mathrm{w} / \mathrm{w})$ were carried out in a Büchi 290 194 Mini Spray Dryer (BÜCHI Labortechnik AG, Flawil, Switzerland). The concentration of the 195 excipients were with respect to the mass of the nanoparticles before spray drying. The 196 following operating conditions were used based on pilot experiments: inlet temperature 120 $197{ }^{\circ} \mathrm{C}$, approximate outlet temperature $65-70{ }^{\circ} \mathrm{C}$, the drying airflow $600 \mathrm{~L} / \mathrm{h}$, aspiration rate $198100 \%\left(35 \mathrm{~m}^{3} / \mathrm{h}\right)$, the nozzle diameter was a $0.1 \mathrm{~mm}$ and the liquid feed rate was set to 5 $199 \mathrm{ml} / \mathrm{min}$. Each preparation were carried out in triplicate. Following spray drying, the powders 200 were collected from the lower part of the cyclone and the collecting vessel, stored in tightly 201 sealed glass vials under vacuum at room temperature.

202 2.4. Characterization of spray-dried BSA-Api-NPs

203

\subsubsection{Determination of residual moisture}


The moisture content of the spray-dried powders was measured by using Karl Fischer titration (Metrohm 758 KFD Titirino, Metrohm AG, Lichtenstein, Switzerland). For that purpose approx. $100 \mathrm{mg}$ of the product was analysed and the instrument was previously calibrated with $10 \mu$ distilled water. The evaluation was conducted in triplicate and the standard deviation calculated.

\subsubsection{Fourier-Transform Infrared Spectroscopy (FT-IR)}

FT-IR spectra of BSA-Api spray-dried samples were evaluated using a PerkinElmer

Spectrum 100 FT-IR spectrometer equipped with Universal ATR (Attenuated Total

Reflectance) accessory (PerkinElmer Inc., Waltham, USA). Approximately $2 \mathrm{mg}$ of the solid

samples were placed between the plate and the probe. The spectra were recorded with 3 scans,

214 in the frequency range between $4000-600 \mathrm{~cm}^{-1}$ and with a resolution of $4 \mathrm{~cm}^{-1}$ at room temperature. The data were analyzed using the PerkinElmer Spectrum Express software.

\subsubsection{X-ray powder diffraction (XRPD)}

XRPD diffractograms were obtained using an X-ray diffractometer (MiniFlex600 Rigaku Corporation, Tokyo, Japan). The analyses were performed at room temperature and the samples were scanned from $2^{\circ}$ to $40^{\circ} 2 \theta$ using a scanning speed of $2 \%$ min with a step size of $0.05^{\circ}$.

\subsubsection{Differential scanning calorimetry (DSC) analysis}

The spray-dried formulations were characterized by DSC (DSC Q2000 module; TA Instruments, New Castle, UK) which was calibrated using indium. Samples (3-5 mg) were weighed accurately and analyzed in sealed and pierced aluminium hermetic pans (TA Instruments). The pans were equilibrated at $25^{\circ} \mathrm{C}$ and then heated at a rate of $10{ }^{\circ} \mathrm{C} / \mathrm{min}$ in a range of $50-400{ }^{\circ} \mathrm{C}$.

\subsubsection{Aerosol particle size analysis and redispersibility in water}



diffractometer (Sympatec GmbH System-Partikel-Technik, Clausthal-Zellerfeld, Germany). The powders were dispersed by compressed air ( $4-5$ bar) into the measuring zone of the laser beam. The optical lens $(0.45-87.5 \mu \mathrm{m}$ size range $)$ focused onto the detector to collect the diffracted light for calculation of size distribution. The values of 10 th $\left(\mathrm{D}_{10}\right), 50$ th $\left(\mathrm{D}_{50}\right)$ and 90th $\left(\mathrm{D}_{90}\right)$ of the cumulative particle size distribution are generated. Samples were measured 234 in triplicate.

235 The particle size was also determined after spray drying. $5 \mathrm{mg}$ dry powder of each 236 formulation could be easily redispersed in $5 \mathrm{ml}$ distilled water and the particle size was 237 determined without any further dilution by the above mentioned Zetasizer Nano ZS 238 instrument (Malvern Instruments Ltd., Worcestershire, UK).

\subsubsection{Solubility and Drug release studies of BSA-Api formulations}

The solubility of BSA-Api formulations were determined in PBS buffer (pH 7.4) and

241 in modified simulated lung fluid (mSLF, $\mathrm{pH}$ 7.4) which contained $0.02 \%(\mathrm{w} / \mathrm{v})$ DPPC was

242 prepared according to Son and McConville ${ }^{30} .50 \mathrm{mg}$ of samples of spray dried powders were 243 added to $100 \mathrm{~mL}$ solvent and shaken $(150 \mathrm{rpm})$ for $2 \mathrm{~h}$ at $37^{\circ} \mathrm{C}$. At predetermined time points

$2441 \mathrm{~mL}$ of samples were taken from each dissolution media and replaced with the same volume

245 of fresh medium. All of the samples were diluted with $1 \mathrm{ml}$ methanol and filtered with 246 Amicon ${ }^{\circledR}$ Ultra Centrifugal filters (30K, Merck Millipore, Merck KGaA, Germany) prior to 247 the injection and the amount of apigenin was determined by HPLC-UV method.

248 The in vitro drug release study of the three formulations were conducted with Franz cell 249 apparatus. The mSLF was used as dissolution media and $0.45 \mu \mathrm{m}$ cellulose acetate membrane 250 filter (Sartorius AG, Goettingen, Germany) was applied. Briefly, an accurately weighed 251 amount $(10 \mathrm{mg})$ of spray dried nanoparticles of each formulations were scattered onto the 252 membrane which was previously wetted with the dissolution media for 1 hour. $1 \mathrm{ml}$ of 
253 samples were withdrawn at various time intervals for 5 hours and replaced with fresh 254 dissolution medium. After the measurement, membrane was rinsed with $2 \mathrm{ml}$ of $\mathrm{MeOH}$ and 255 the drug content of the possibly remained powders was determined. The sample preparation 256 and the measurement was the same as mentioned above. The cumulative amount of apigenin 257 release over the time was plotted for each formulations. All measurements were performed in 258 triplicate.

\section{$259 \quad$ 2.4.7. Aerosol delivery of BSA-Api formulations}

260 In vitro aerodynamic performance of BSA-Api formulations was assessed using the 261 next generation impactor (NGI; Copley Scientific Ltd., Nottingham, UK), connected 262 sequentially to a low capacity pump via the critical flow controller (Model LCP5; Copley 263 Scientific Ltd., Nottingham, UK). During the measurement the pump was operated at air flow 264 rate of $60 \mathrm{~L} / \mathrm{min}$ for $4 \mathrm{~s}$. The $3 \times 10 \mathrm{mg}$ powder aliquots from each formulation were loaded 265 manually into gelatine capsules (size 3) and placed into the inhaler device (Cyclohaler ${ }^{\circledR}$, 266 Pharmachemie, London, UK) which was connected to the NGI via an airtight rubber adaptor 267 and a stainless steel USP throat. The NGI stages were assembled with an induction port, a 268 pre-separator and a filter was placed in the final stage. Prior to the impaction, the collection 269 plates were uniformly coated with $1 \mathrm{ml}$ of $1 \%$ silicone oil in N-hexane solution and allowed 270 to dry leaving a thin film of silicone oil on the plate surface in order to prevent the re271 entrainment of the particles and the pre-separator was filled with $15 \mathrm{ml}$ DMSO:MeOH $272(50: 50 \%, v / v)$ mixture. After the deposition of the powders in the NGI, the amount of each 273 formulation was cumulatively collected onto silicone-coated plates for each of the stages. The 274 inhaler, mouth piece, induction port, pre-separator and the collection plates were rinsed with 275 DMSO:MeOH (50:50\%, v/v) mixture, collected in volumetric flasks (10 or $25 \mathrm{ml})$ and made 276 up to volume. The samples were determined by using HPLC method as described previously. 277 To characterize the aerosol performance the following parameters were calculated based on 
278 the drug mass of each fraction: emitted dose (ED, \%): the percentage of the entire dose

279 depositing from the mouthpiece of the inhaler device and recovered dose (RD, \%): the total 280 recovered drug mass. The fine particle fraction $(\mathrm{FPF},<4.46 \mu \mathrm{m})$ is defined as the percentage 281 of the emitted dose which deposited from the Stage 2-7 and the micro orifice-collector 282 (MOC). The mass median aerodynamic diameter (MMAD) and geometric standard deviation 283 (GSD) were calculated from the inverse of the standard normal cumulative mass distribution 284 against the natural logarithm of the effective cut-off diameter of the respective stages. All 285 measurements were carried out in triplicate.

286 2.4.8. Particle morphology

287 Morphology of Api powder and spray-died nanoparticles was examined using 288 scanning electron microscopy (SEM) analysis. The dry powder of the formulations was 289 placed on the sample holder using double adhesive tape and gold coating ( $20 \mathrm{~nm}$ thickness) 290 was applied. Examinations were performed by FEI Inspect ${ }^{\mathrm{TM}} \mathrm{S} 50$ (Hillsboro, Oregon, USA) 291 scanning electron microscope at $20.00 \mathrm{kV}$ accelerating voltage. Original magnifications were $2928000 x, 10,000 x$ and $20,000 x$ with accuracy of $\pm 2 \%$.

$293 \quad$ 2.5. Antioxidant activity

294 The antioxidant activities of the prepared spray-dried formulations were compared to 295 the pure Api in order to investigate the effectiveness of the formulation. The free radical 296 scavenging activity was measured by using $\mathrm{DPPH}^{\bullet}$ method as described previously ${ }^{31}$ with 297 slight modifications. Methanolic stock solution of $0.1 \mathrm{mM} \mathrm{DPPH}$ reagent was freshly 298 prepared and protected from light. Standard curve was plotted between the DPPH 299 concentration (0.01-0.1 $\mathrm{mM}$ ) and absorbance, the linear relationship was calculated 300 graphically. $1 \mathrm{ml}$ of $\mathrm{MeOH}$ was added to the BSA-Api-NPs and the concentration of Api were 301 the same in each sample for the comparability. Thereafter $2 \mathrm{ml}$ of $0.06 \mathrm{mM} \mathrm{DPPH}^{\bullet}$ reagent 302 was added to the samples, vortex mixed for 10 seconds and protected from light. The 
absorbance at $517 \mathrm{~nm}$ was determined with spectrophotometer (UV-Vis spectrophotometer, Metertech SP-8001, Metertech Inc., Taipei, Taiwan) in every 15 minutes until the steady state (when no further discoloration could be observed). The addition of samples resulted decrease in the absorbance of $\mathrm{DPPH}^{\cdot}$ due to the scavenging activity of Api. The exact concentration of the free radical was calculated using the standard curve. To calculate the inhibition of the free radical DPPH${ }^{\bullet}$ the following equation (Eq.3.) was used:

$$
I(\%)=\frac{A_{0}-A_{s}}{A_{0}} \times 100
$$

Where I $(\%)$ is the inhibition in percent, $\mathrm{A}_{0}$ is the absorbance of the $\mathrm{DPPH}^{\bullet}$ solution and $\mathrm{A}_{\mathrm{s}}$ is the absorbance of the sample. All measurements were carried out triplicate and the data were expressed as the mean value $\pm \mathrm{SD}$.

\section{RESULTS AND DISCUSSION}

\subsection{Characterization of BSA-Api-NPs}

\subsubsection{Size, zeta potential and drug content}

Albumin is a natural protein that has been widely used as a macromolecular carrier for many drugs with low water solubility. Several techniques are available to prepare albumin nanoparticles including desolvation (coacervation), nab (nanoparticle albumin bound)technology and self-assembly ${ }^{14}$. In this study the BSA-Api-NPs were prepared by using modified nab-technology with ultrasonication. The achieved mean particle size of three samples was $376 \pm 7.824 \mathrm{~nm}$ with a polydispersity index of $0.285 \pm 0.01$. The size of albumin NPs less than $500 \mathrm{~nm}$ could localize effectively in the lung. The PDI value indicated narrow particle size distribution and the uniformity of the nanoparticles. The zeta potential was -19.20 $\pm 0.818 \mathrm{mV}$. The higher the zeta potential, the more stable the formulation is, less aggregation occurs $^{32}$. The EE was determined to be $82.61 \pm 4.56 \%$ and the DL was $7.51 \pm 0.415 \%$. Therefore these results confirmed the high encapsulation efficiency of apigenin by BSA-NPs and it can be attractive tool in encapsulation flavonoids for delivery. Similar data were found 
328 in the literature when encapsulating flavonoids into albumin nanoparticles. Human serum

329 albumin-bound curcumin nanoparticles resulted $7.2 \pm 2.5 \%$ loading efficiency ${ }^{33}$ and

330 scutellarin-loaded bovine serum albumin nanoparticles possess $64.46 \% \mathrm{EE}$ and $6.73 \% \mathrm{DL}^{34}$.

\section{$331 \quad 3.1 .2$. Fluorescence spectroscopy}

332 The phenomenon of fluorescence quenching can result from various inter and 333 intramolecular interactions such as energy transfer, conformational changes, complex 334 formation (static quenching) or collisional interaction (dynamic quenching). During static 335 quenching the quencher forms a stable non-fluorescent complex with the fluorophore, 336 however, during dynamic quenching it collides with the fluorophore and facilitates non337 radiative transitions to the ground $\operatorname{state}^{35}$. Therefore quenching of the intrinsic fluorescence of 338 the two tryptophan residues (Trp-134 and Trp-212) of BSA can offer information about the 339 changes in molecular microenvironment of these fluorophores, located in domain I and II, 340 respectively. Trp-134 residue is located close to the protein surface in a hydrophilic 341 environment, while Trp-212 is within a protein pocket which is hydrophobic (subdomain II 342 A). The Trp-214 in human serum albumin (HSA) is located similarly to Trp-212 in BSA ${ }^{36-38 .}$

343 The quenching effect of Api on fluorescence intensity of serum albumins (BSA and HSA) has 344 been studied previously ${ }^{36,39-42}$ but there is no data related to its behavior in a nanoparticulate 345 system. Studies have shown that the increasing concentration of Api resulted in a decrease in 346 the fluorescence emission intensity of serum albumin solutions. This was mainly attributed to 347 complex formation (static quenching), however, it could be dynamic quenching at higher Api 348 concentrations $^{42}$. Nevertheless, all studies concluded that Api most likely binds to the sub349 domain IIA of Site I side with electrostatic and hydrophobic interactions, through which H350 bonds and non-radiative energy transfer can occur. The binding could affect the conformation 351 of Trp micro-region but the secondary structure of serum albumin is not altered ${ }^{36,} 39,41$. 
352 However, the $\mathrm{pH}$ and ionic concentrations (e.g. $\mathrm{NaCl}$ ) can affect the fluorescence quenching 353 on the binding parameters of apigenin to $\mathrm{BSA}^{43}$.

354 Figure 2 demonstrates the fluorescence emission spectra of BSA solution, BSA-NPs 355 and BSA-Api-NPs. The fluorescence intensity of BSA-NPs decreased slightly compared to 356 BSA solution with no obvious shift of the maximum position at $350 \mathrm{~nm}$. It was probably due 357 to the conformational changes of the protein. The significantly lower emission intensity of 358 BSA-Api-NPs indicates that Api could quench the fluorescence of BSA which is also 359 reflected on the 3D projections (Figure 3). All of these findings indicate that Api binds to the 360 Trp region (Trp-212, subdomain II A) but the spectral maximum was not affected therefore 361 hydrophobicity and polarity of the fluorophore residues are not altered. It was concluded that 362 Api can be bound to the Trp region of serum albumin nanoparticles similarly to the solutions.

\subsection{Characterization of spray-dried BSA-Api-NPs}

\subsubsection{Determination of residual moisture}

Moisture content is mainly influenced by the spray drying conditions. Increased heat energy availability provided by regulating inlet air temperature and aspirator capacity allows more efficient drying, thus resulting in the lower moisture content demonstrated. However, degradation of heat sensitive materials such as proteins may occur; therefore inlet air temperature should be kept below $120^{\circ} \mathrm{C}^{44}$. The water content is also affected by the type of excipients and the ratio with the nanoparticles ${ }^{45}$. Moisture content is an important factor that can significantly influence the aerodynamic properties of aerosols. It can change the surface of particles, promote aggregation and influence the crystallinity of the spray-dried samples ${ }^{44}$. In this study, the residual water content was determined by using Karl Fisher titration. All formulations had relatively low moisture content which followed the rank order of L-leucine $(4.11 \pm 0.21 \%, \mathrm{w} / \mathrm{w})<\operatorname{excipient-free}(4.55 \pm 0.49 \%, \mathrm{w} / \mathrm{w})<$ lactose $(5.8 \pm 0.36 \%, \mathrm{w} / \mathrm{w})$ containing products. These results demonstrate that the optimized outlet air temperature 
377 (around $65^{\circ} \mathrm{C}$ ) was suitable for serum albumin. The L-leucine containing formulation had the

378 lowest water content due to the low hygroscopic behavior of this amino acid ${ }^{46,47}$. The low

379 moisture content can potentially improve the flowability and consequently enhance lung 380 deposition due to reduced aggregation as expected. Storage conditions are also important, e.g.

381 the spray-dried amorphous lactose particles could transform into crystals easily in humidity 382 above $30 \%{ }^{48}$.

\section{$383 \quad 3.2 .2$. Fourier-Transform Infrared Spectroscopy (FT-IR)}

384 FTIR analysis allows a quick and efficient identification of the compounds and by 385 their functional groups and bond vibrations. In the spectrum of raw Api, the following 386 characteristic regions were observed: $2710-2580 \mathrm{~cm}^{-1} \mathrm{O}-\mathrm{H}$ bond, $1730-1680 \mathrm{~cm}^{-1} \mathrm{C}=\mathrm{O}$ stretch 387 and $1450-1380 \mathrm{~cm}^{-1} \mathrm{C}-\mathrm{H}$ bend. A broad peak observed at $3300 \mathrm{~cm}^{-1}$ can be attributed to O-H 388 stretching and those bands at $1600-1400 \mathrm{~cm}^{-1}$ (C-C stretch in ring) and 900-675 $\mathrm{cm}^{-1}(\mathrm{C}-\mathrm{H}$ 389 'oop') can be assigned to the aromatic group (Figure 4 A). In the spectrum of BSA protein, 390 the amide I band at $1635 \mathrm{~cm}^{-1}$ (mainly $\mathrm{C}=\mathrm{O}$ stretch) and amide II band at $1530-1500 \mathrm{~cm}^{-1}(\mathrm{C}-$ $391 \mathrm{~N}$ stretching and $\mathrm{N}-\mathrm{H}$ bend) can be seen. The medium broad peak at $3276 \mathrm{~cm}^{-1}$ corresponds to 392 bonded N-H stretch of amide and a smaller band at $1057 \mathrm{~cm}^{-1}$ is the C-N stretch of aliphatic 393 amine. In the spectra of the excipients-free formulation, the characteristic amide bands of 394 BSA can be seen and peak at $830 \mathrm{~cm}^{-1}$ indicating the presence of Api (aromatic) which is an 395 indirect confirmation of Api encapsulation on BSA-NPs. Conformational changes can be 396 suggested due to the lack of the peak of aliphatic amine.

397 The spectra of raw Api, BSA, lactose and lactose containing product are displayed on Figure $3984 \mathrm{~B}$. In the spectra of lactose there is also a broad band around $3300 \mathrm{~cm}^{-1}$ indicating the 399 stretching vibration of hydroxyl group. A weak band at $1654 \mathrm{~cm}^{-1}$ is the bending vibration of 400 the crystalline water and peaks at $1200-1070 \mathrm{~cm}^{-1}$ demonstrate the stretching vibration of C401 O-C in the glucose and galactose. The spectrum of amorphous lactose has the less number and 
402 defined peaks and therefore it could be distinguished from the crystalline spectrum ${ }^{49}$. The 403 characteristic broad band at $3300 \mathrm{~cm}^{-1}$ in the spectrum of spray-dried product could originate 404 from the residual water content that is further supported by the Karl Fischer titration data 405 (lactose containing product had the highest water content). Similarly to the spectrum of 406 excipients-free formulation, the amide bands of BSA and a small peak of Api could be 407 observed. The peaks at $1200-1070 \mathrm{~cm}^{-1}$ demonstrate the lactose content and the amorphous 408 state could be assumed.

409 Functional groups of L-leucine lead to its characteristic spectrum (Figure 4 C). The strong 410 band in the region of $2970-2910 \mathrm{~cm}^{-1}$ can be accounted for the aliphatic C-H stretching. The 411 bonded N-H stretch is present in the region of $2600-2450 \mathrm{~cm}^{-1}$. The $\mathrm{NH}_{2}$ bending and the C$412 \mathrm{~N}$ skeletal vibration appear in the regions of $1595-1550 \mathrm{~cm}^{-1}$ and $1250-1020 \mathrm{~cm}^{-1} 50$. The 413 presence of BSA characteristic peaks on the spray-dried formulation could be mainly 414 observed with and a small peak of Api but the major characteristic peaks of L-leucine are 415 obscured.

\section{$416 \quad 3.2 .3$. X-ray powder diffraction (XRPD)} XRPD is considered to be the most accurate method to study crystalline structure ${ }^{51}$. The combined XRPD diffractograms of Api and spray-dried formulations are presented in Figure 5. The characteristic narrow diffraction peaks of Api are due to the crystalline state of the commercially available material. In comparison, broad diffuse peaks could be observed in 421 the diffractograms of the spray-dried formulations suggesting the amorphous state of BSA422 Api-NPs. The observed XRD patterns of spray-dried L-leucine and lactose were consistent 423 with literature ${ }^{48,52,53}$.

\section{3.2.4. Differential scanning calorimetry analysis (DSC)}

425 The DSC curves of raw Api, excipents, physical mixtures and spray-dried 426 formulations were studied to examine crystallinity. As seen in Figure 6, there is only one 
427 sharp endothermic peak at $360{ }^{\circ} \mathrm{C}$ indicating the melting point of raw Api; no impurities were 428 observed. Drug free albumin exhibited two broad peaks with onset values of $220{ }^{\circ} \mathrm{C}$ and 310 $429{ }^{\circ} \mathrm{C}$. The evaporation of residual water occurred at $50-120{ }^{\circ} \mathrm{C}$. The melting point of Api on the 430 thermograms of raw material and physical mixtures corresponds to the crystalline habitus. In 431 the thermograms of physical mixtures on Figure $6 \mathbf{B}$ the endothermic peak at $140{ }^{\circ} \mathrm{C}$ 432 indicating the crystalline lactose ${ }^{54}$ and the sublimation of L-leucine crystals occurred at 200$433230{ }^{\circ} \mathrm{C}$ (Figure 6 C) ${ }^{55}$. However, in each spray-dried formulation the absence of endotherms 434 confirms the loss of crystallinity. No peak could be observed around $360{ }^{\circ} \mathrm{C}$ indicating that 435 Api is in amorphous state due to the spray drying process which is in agreement with the 436 XRPD diffractograms. The amorphous form generated may result higher solubility of the 437 powders and dissolution of apigenin in lung fluids.

\subsubsection{Aerosol particle size analysis and redispersibility in water}

Dry powder formulations of BSA-Api-NPs were prepared with the aim of studying the 440 influence of excipients on the particle size and aerodynamic behavior. The deposition of 441 aerosols is significantly affected by particle size which should be small enough to pass 442 through the upper airways and large enough to avoid exhalation ${ }^{56}$. Gravitational 443 sedimentation is the main driving force for deposition of a nanoparticulate system in the lung 444 due to the formation of aggregates in the micrometer size range. Particle geometry and surface 445 properties also play a significant role in reaching the bronchioles ${ }^{22,32}$. It is well known that 446 particles can be deposited efficiently deeper in the lung if their aerodynamic diameter is in the 447 range of 1-5 $\mu \mathrm{m}$ and only those with $1-3 \mu \mathrm{m}$ can reach the respiratory zone ${ }^{57}$. Particles, larger 448 than $5 \mu \mathrm{m}$ tend to deposit in the oropharynx and the mucociliary clearance plays a role in 449 clearing the particles towards the pharynx. However, very small particles, less than $1 \mu \mathrm{m}$ are 450 usually exhaled because of the low inertia ${ }^{58,59}$. Mucociliary clearance is the part of the natural 451 defense mechanism of the lung as well as the phagocytosis of macrophages in the alveolar 
452 region. The aerosol particle size was determined by Sympatec HELOS laser diffractometer

453 (Table 1). The excipient-free and lactose containing products have similar sizes while spray 454 drying with L-leucine produced the smallest particles $\left(\mathrm{D}_{50}=2.473 \mu \mathrm{m}\right)$. In all cases, the 455 particle size could ensure the highest probability of delivery of apigenin into the respiratory 456 zone.

457 Following the re-dispersion of spray powders formulations in distilled water, the size of the 458 particles was preserved in the nanometer size range: without excipient $(358.9 \pm 5.3 \mathrm{~nm}$, PDI: $4590.315 \pm 0.013)$, lactose $(366.1 \pm 4.8 \mathrm{~nm}$, PDI: $0.382 \pm 0.014)$ and L-leucine $(343.7 \pm 2.9$, PDI: $460 \quad 0.316 \pm 0.011)$ containing products. The spray drying has no significant effect on the average 461 size of the particles suggesting the deposition of apigenin containing nanoparticles in the lung 462 fluid. Moreover, the excipients did not affect adversely the particle size.

\subsubsection{Solubility and Drug release studies of BSA-Api formulations}

The solubility of apigenin in nanoparticles was investigated in PBS buffer and mSLF (Figure 7 A). The results showed that the solubility was slightly increased in mSLF media (82-98\% within 5 minutes), however, it was high in PBS buffer as well (79-95\% within 5 minutes). These data indicated that the solubility of apigenin could be highly enhanced by BSA nanoparticles in aqueous medium. Nevertheless, the dispersibility enhancers could play a role in the solubility. In case of excipient free formulation, $91 \%$ of the encapsulated apigenin was dissolved in mSLF within 5 minutes. Formulation prepared with lactose increased the solubility rate up to $98 \%$, however, it was slower ( $82 \%)$ when using L-leucine and completed within 2 hours. These results could be attributed to the solubility of the excipients themselves: lactose has very good water solubility, but L-leucine possess a low solubility in water .

The apigenin release from the spray dried BSA-Api NPs was investigated with Franz cell apparatus. It is a well known device for the dissolution of semisolid dosage forms and 
477 approved by the USP (United States Pharmacopeia). However, there is no standardized 478 method for inhaled powders, Franz cell could be one of the alternative choices due to 479 simulating the diffusion controlled air-liquid interface of the lung. On the contrary, it has 480 some limitations such as small air bubbles under the contact area of membrane to dissolution 481 medium, wide range of standard deviation or the recovery usually around maximum $90 \%{ }^{61}$. 482 Based on the solubility measurements, mSLF was applied. The cumulative dissolution curves 483 of the prepared formulations are shown in Figure 7 B. As expected, the dissolution was 484 affected by the co-spray dried excipients. Lactose containing product resulted the fastest and 485 highest apigenin release due to the excellent water solubility. This enhancement of the 486 dissolution is supported by previously published data ${ }^{62}$. In contrast, the dissolution rate was 487 decreased when L-leucine was applied. The coating layer of L-leucine slowed down the 488 dissolution of apigenin which could be well observed in the dissolution curve. The low water 489 solubility of L-leucine is able to hinder the dissolution of the drug which was published 490 previously $^{60}$. These results suggest that the excipients play an important role in the solubility 491 and the dissolution as well.

$492 \quad 3.2 .7$. Aerosol delivery of BSA-Api formulations

493 Particles can be taken up by alveolar macrophages which influences the therapeutic 494 outcome. Those nanoparticles which are soluble and above $200 \mathrm{~nm}$ are able to escape from 495 the macrophages therefore exhibit sustained therapeutic effect ${ }^{63}$. The lung deposition and 496 therefore the efficacy of the inhaled therapeutics are governed by their aerosol properties ${ }^{56}$. 497 Manufacturing respirable nanoparticles could be produced by aggregation in the favorable 498 size range or their incorporation into microparticles $(1-5 \mu \mathrm{m})^{26}$. Lactose monohydrate is a 499 well-known, traditional carrier for improving the performance of inhaled products; however, 500 it is influenced by physicochemical properties and interaction with the active ingredient ${ }^{64,65}$. 501 It is the only FDA approved carrier and has also been shown to be a potential excipient for 
502 protein encapsulation ${ }^{27,65}$. Recently, novel materials such as specific amino acids have been 503 developed for pulmonary formulations ${ }^{26}$ and L-leucine is one of the most effective 504 dispersibility enhancer among them ${ }^{47}$. Previous studies proved that $5 \%(\mathrm{w} / \mathrm{w})$ L-leucine 505 improved the aerosol performance of raw naringin ${ }^{66}$ and inclusion up to $15 \%(\mathrm{w} / \mathrm{w})$ L-leucine 506 resulted higher ED and FPF of powder formulation of gentamicin ${ }^{46}$.

507 In this study in vitro aerosol properties of three different dry powders formulations 508 were evaluated using the NGI which is regarded as an optimal instrument for analysis of 509 aerodynamic behavior of aerosol formulations for pulmonary drug delivery ${ }^{67}$ according to 510 European and US Pharmacopeias. The obtained data and deposition pattern are presented in 511 Table 2 and in Figure 8. It can be seen that more than $90 \%$ of apigenin could be recovered 512 from the NGI which is in the acceptable pharmacopeia range (75-125\%). The ED ranged 513 between $91-96 \%$ indicating good flowability and high dispersibility of the powders. L-leucine 514 containing formulation had the highest ED as it could improve significantly the flowability of 515 the powders ${ }^{47,53}$. Figure 8 shows the amount of Api deposited on the throat, device and 516 stages 1-7 expressed as a percentage of the total amount of recovered powder. All formulation 517 exhibited increased deposition in Stage 2 - 4 indicating enhanced drug delivery to the alveolar 518 regions. As expected, improved aerosol performance and deposition (Stage 3 and 4) could be 519 observed when L-leucine was used an as excipient. The FPF is one of the key parameters in 520 aerosol delivery and should be as high as possible ${ }^{68}$. In this study, the FPF values ranged 521 between $58-66 \%$, suggesting that the particles could be delivered into the peripheral regions. 522 Spray drying of nanoparticles in the presence of L-leucine resulted higher FPF value (66\%) 523 due to the improved surface properties and morphology of the particles ${ }^{65}$. In general, MMAD 524 values $<5 \mu \mathrm{m}$ are for pulmonary lung delivery and between 2-3 $\mu \mathrm{m}$ are optimal for deep lung 525 deposition ${ }^{56}$. In each cases, the calculated mass median aerodynamic diameter (MMAD) data 526 were in agreement with the physical diameter size of the particles measured by laser 
527 diffractometer. The data obtained $(<5 \mu \mathrm{m})$ support good dispersibility of the particles into the

528 lower airways and the deep lung. Therefore local delivery to the alveoli could be assured by

529 both excipient-free and lactose formulations generated (MMAD $3.2 \mu \mathrm{m}$ and $3.1 \mu \mathrm{m}$ ).

530 Moreover, formulation with L-leucine (MMAD $2.1 \mu \mathrm{m})$ would be more optimal for deep lung

531 deposition. The size distribution of an aerosol is described best by GSD ${ }^{69}$. Based on the GSD

532 data obtained, the L-leucine containing formulation had the narrowest size distribution (1.8

$533 \mu \mathrm{m})$ but that of the others was also in the acceptable range $(<3 \mu \mathrm{m})$.

534 The overall values demonstrate that the particles of each dry powder nanoparticle formulation

535 are in the favorable aerodynamic size range, possess good dispersibility properties and 536 particle deposition. Therefore BSA-NPs is an attractive delivery system for pulmonary drug

537 delivery. We demonstrated that L-leucine improved better the aerosolization properties of

538 BSA-Api-NPs than lactose monohydrate. Therefore it can be concluded that the use of 539 excipients influence the aerosol performance of nanoparticles.

$540 \quad$ 3.2.8. Particle morphology

541 SEM analysis was conducted to investigate the morphology of the powders (Figure 9

542 A and B). It is well known that the morphology of the particles is strongly affected by the

543 solubility of the components and the nature of the excipients ${ }^{46,47}$. The commercially available

544 Api was a crystalline powder featuring needle-shaped crystals. The excipient-free spray-dried

545 nanoparticles exhibited spherical shape and smooth or wrinkled surface. Particles of lactose

546 containing product had raisin-like surface and some of the particles were larger in accordance

547 with the laser diffraction particle size analysis. Powders prepared with L-leucine comprised

548 small and collapsed particles with strongly corrugated surface. The low aqueous solubility of

549 L-leucine leading to a shell on the surface of the droplet which interfere with the diffusion of

550 water therefore corrugated particles could form. This outcome was consistent with previous

551 observations ${ }^{46,53,70}$. Corrugated surface improves the dispersibility of the dry powder 
552 formulations and enhance respirability due to the reduced interparticulate cohesion (Van der 553 Waals forces) which is beneficial for particles intended for inhalation ${ }^{71}$.

$554 \quad 3.3$. Antioxidant activity

555 Owing to its reproducibility and comparability, the $\mathrm{DPPH}^{\circ}$ assay is an established 556 method for investigating the antioxidant properties of natural compounds. Due to the $\mathrm{H}$ 557 donating ability of the antioxidants, a stable reduced DPPH-H molecule can form. The 558 reaction can be seen visually and the detection can be carried out using UV-Vis 559 spectrophotometer ${ }^{72,73}$. Previous studies confirmed that Api is able to scavenge the DPPH 560 free radial even in nanoscale delivery formulation ${ }^{74,75}$. In general, the scavenging activity is 561 influenced by concentration and structural features like hydrogen donating ability, position 562 and the degree of hydroxylation ${ }^{76,77}$. In order to calculate the exact concentration of 563 remaining $\mathrm{DPPH}^{*}$ in the samples a calibration curve was plotted with $\mathrm{R}^{2}=0.9999$. The time 564 required to reach the steady state was estimated to be 120 minutes, and the slow reaction 565 kinetic of Api has been reported ${ }^{74}$. The discoloration of the deep purple DPPH ${ }^{\bullet}$ free radical 566 indicate the antioxidant properties of free and encapsulated Api. The inhibition of free 567 radicals by the prepared spray-dried formulations were compared to the empty BSA-NPs, 568 methanolic Api solution and "empty" nanoparticles (Figure 10). It can be seen that the free 569 and encapsulated Api have similar scavenging activity, moreover, the spray drying did not 570 result in the loss of scavenging activity. It has been reported that serum albumin is a 571 physiological circulating antioxidant in the body ${ }^{78}$ which is confirmed by the inhibition 572 capacity of the empty BSA-NPs observed. Similar results were reported when encapsulating 573 rutin and keampferol ${ }^{79}$ or quercetin ${ }^{17}$ where the antioxidant activity of the flavonoids are 574 retained by BSA. It can be concluded that the antioxidant activity of Api is preserved, 575 moreover, slightly enhanced by the BSA. 
577 In this study novel apigenin containing albumin nanoparticles were prepared for 578 inhalation against lung injury caused by oxidative stress. Apigenin was recently classified as a

579 BCS II. drug with prominent antioxidant and anti-inflammatory properties in the lung. The 580 obtained results confirmed that incorporation of apigenin into the biocompatible albumin 581 nanoparticles resulted high encapsulation efficiency therefore it could be an attractive tool for 582 the delivery. Moreover, the spray dried nanoparticles possess good ability to re-disperse in 583 aqueous media and size of the particles was preserved in the nanometer size range. The 584 influence of dispersibility enhancers on the physicochemical properties and in vitro 585 pulmonary deposition were investigated and compared to the excipient-free formulation. The 586 obtained in vitro pulmonary depositions proved that the developed BSA-NP dry powders are 587 potentially able to carry apigenin deep in the lung, reaching the respiratory zone. The use of 588 novel excipient amino acid L-leucine resulted enhanced aerodynamic properties over the 589 traditional lactose monohydrate, indicating that the nature of the excipients and morphology 590 of the particles play a significant role in the formulation of nanoparticles for pulmonary 591 delivery. In addition, the solubility and dissolution characteristics of apigenin from 592 nanoparticles were determined in mSLF dissolution media, the co-spray dried excipients 593 played an important role. The dissolution rate was increased by the water soluble lactose and 594 decreased by L-leucine, which has low water solubility. Therefore the use of excipients should 595 be taken into consideration, may not required in case of albumin nanoparticles. We further 596 confirmed that the antioxidant activity is retained, thus the potential of albumin nanoparticles 597 as an effective pulmonary delivery system for flavonoids such as apigenin.

\section{ACKNOWLEDGEMENTS}

599 The authors gratefully acknowledge to Róbert Kovács for providing the SEM pictures at 600 Budapest University of Technology and Economics.

\section{Author Disclosure Statement}


602 The authors declare that there are no conflicts of interest.

603 


\section{REFERENCES}

605 1. Chow C-W, Herrera Abreu MT, Suzuki T, and Downey GP. Oxidative Stress and 606 Acute Lung Injury. Am J Respir Cell Mol Biol. 2003;29:427-431.

607 2. Chen XW, Serag ES, Sneed KB, and Zhou SF. Herbal bioactivation, molecular targets 608 and the toxicity relevance. Chem Biol Interact. 2011;192:161-176.

609 3. Patel D, Shukla S, and Gupta S. Apigenin and cancer chemoprevention: progress, 610 potential and promise (review). Int J Oncol. 2007;30:233-245.

611 4. Kim SJ, Jeong HJ, Moon PD, Lee KM, Lee HB, Jung HJ, Jung SK, Rhee HK, Yang 612 DC, Hong SH, and Kim HM. Anti-inflammatory activity of gumiganghwaltang 613 through the inhibition of nuclear factor-kappa B activation in peritoneal macrophages. 614 Biol Pharm Bull. 2005;28:233-237.

615 5. Kowalski J, Samojedny A, Paul M, Pietsz G, and Wilczok T. Effect of apigenin, 616 kaempferol and resveratrol on the expression of interleukin-1beta and tumor necrosis 617 factor-alpha genes in J774.2 macrophages. Pharmacol Rep. 2005;57:390-394.

618 6. Lee J-H, Zhou H, Cho S, Kim Y, Lee Y, and Jeong C. Anti-inflammatory mechanisms 619 of apigenin: inhibition of cyclooxygenase-2 expression, adhesion of monocytes to 620 human umbilical vein endothelial cells, and expression of cellular adhesion molecules. 621 Archives of Pharmacal Research. 2007;30:1318-1327.

622 7. Chen L and Zhao WEI. Apigenin protects against bleomycin-induced lung fibrosis in 623 rats. Exp Ther Med. 2016;11:230-234.

624 8. Luan RL, Meng XX, and Jiang W. Protective Effects of Apigenin Against Paraquat625 Induced Acute Lung Injury in Mice. Inflammation. 2016; 39:752-758.

626 9. Basios N, Lampropoulos P, Papalois A, Lambropoulou M, Pitiakoudis MK, Kotini A, 627 Simopoulos $\mathrm{C}$, and Tsaroucha AK. Apigenin Attenuates Inflammation in 
628

629

630

631

632

633

634

635

636

637

638

639

640

641

642

643

644

645

646

647

648

649

650

651

Experimentally Induced Acute Pancreatitis-Associated Lung Injury. J Invest Surg. 2015;2:1-7.

10. Patil R, Babu RL, Naveen Kumar M, Kiran Kumar KM, Hegde S, Ramesh G, and Chidananda Sharma S. Apigenin inhibits PMA-induced expression of proinflammatory cytokines and AP-1 factors in A549 cells. Mol Cell Biochem. 2015;403:95-106.

11. Wang J, Liu Y-T, Xiao L, Zhu L, Wang Q, and Yan T. Anti-Inflammatory Effects of Apigenin in Lipopolysaccharide-Induced Inflammatory in Acute Lung Injury by Suppressing COX-2 and NF-kB Pathway. Inflammation. 2014;37:2085-2090.

12. Zhang J, Liu D, Huang Y, Gao Y, and Qian S. Biopharmaceutics classification and intestinal absorption study of apigenin. Int J Pharm. 2012;436:311-317.

13. Ajazuddin and Saraf S. Applications of novel drug delivery system for herbal formulations. Fitoterapia. 2010;81:680-689.

14. Elzoghby AO, Samy WM, and Elgindy NA. Albumin-based nanoparticles as potential controlled release drug delivery systems. J Control Release. 2012;157:168-182.

15. Hu Y-J, Liu Y, Sun T-Q, Bai A-M, Lü J-Q, and Pi Z-B. Binding of anti-inflammatory drug cromolyn sodium to bovine serum albumin. Int J Biol Macromolec. 2006;39:280285.

16. Fasano M, Curry S, Terreno E, Galliano M, Fanali G, Narciso P, Notari S, and Ascenzi P. The extraordinary ligand binding properties of human serum albumin. IUBMB Life. 2005;57:787-796.

17. Fang $\mathrm{R}$, Hao $\mathrm{R}$, Wu $\mathrm{X}$, Li $\mathrm{Q}$, Leng $\mathrm{X}$, and Jing $\mathrm{H}$. Bovine Serum Albumin Nanoparticle Promotes the Stability of Quercetin in Simulated Intestinal Fluid. J Agr Food Chem. 2011;59:6292-6298. 
652 18. He X, Xiang N, Zhang J, Zhou J, Fu Y, Gong T, and Zhang Z. Encapsulation of 653 teniposide into albumin nanoparticles with greatly lowered toxicity and enhanced 654 antitumor activity. Int J Pharm. 2015;487:250-259.

655 19. Muralidharan P, Malapit M, Mallory E, Hayes Jr D, and Mansour HM. Inhalable 656 nanoparticulate powders for respiratory delivery. Nanomedicine: NBM. 2015;11:1189$657 \quad 1199$

658 20. Malcolmson RJ and Embleton JK. Dry powder formulations for pulmonary delivery. $659 \quad$ Pharm Sci Technol To. 1998;1:394-398.

660 21. Stegemann S, Kopp S, Borchard G, Shah VP, Senel S, Dubey R, Urbanetz N, Cittero 661

662

663

664

665

666

667

668

669

670

671

672

673

674

675

676

M, Schoubben A, Hippchen C, Cade D, Fuglsang A, Morais J, Borgström L, Farshi F, Seyfang KH, Hermann R, van de Putte A, Klebovich I, and Hincal A. Developing and advancing dry powder inhalation towards enhanced therapeutics. Eur J Pharm Sci. 2013;48:181-194.

22. Yang W, Peters JI, and Williams Iii RO. Inhaled nanoparticles-A current review. Int J Pharm. 2008;356:239-247.

23. Geiser M. Update on macrophage clearance of inhaled micro- and nanoparticles. $J$ Aerosol Med Pulm Drug Deliv. 2010;23:207-217.

24. Woods A, Patel A, Spina D, Riffo-Vasquez Y, Babin-Morgan A, de Rosales RT, Sunassee K, Clark S, Collins H, Bruce K, Dailey LA, and Forbes B. In vivo biocompatibility, clearance, and biodistribution of albumin vehicles for pulmonary drug delivery. J Control Release. 2015;210:1-9.

25. Swarbrick J. Encyclopedia of Pharmaceutical Technology. Informa Healthcare:London; 2007; 671 - 1434.

26. Mansour $\mathrm{HM}$, Rhee $\mathrm{YS}$, and $\mathrm{Wu} \mathrm{X}$. Nanomedicine in pulmonary delivery. Int $J$ Nanomedicine. 2009;4:299-319. 
677 27. Shoyele SA and Cawthorne S. Particle engineering techniques for inhaled

678

679

680

681

682

683

684

685

686

687

688

689

690

691

692

693

694

695

696

697

698

699

700

701 biopharmaceuticals. Adv Drug Deliv Rev. 2006;58:1009-1029.

28. Sung JC, Pulliam BL, and Edwards DA. Nanoparticles for drug delivery to the lungs. Trends Biotechnol. 2007;25:563-570.

29. Desai NP, Tao C, Yang A, Louie L, Yao Z, Soon-Shiong P, and Magdassi S. Protein stabilized pharmacologically active agents, methods for the preparation thereof and methods for the use thereof. Google Patents, 2004. US6749868 B1

30. Son YJ, McConville JT. Development of a standardized dissolution test method for inhaled pharmaceutical formulations. Int J Pharm. 2009;382:15-22.

31. Hatano T. KH, Yasuhara T., Okuda T. 2 new flavonoids and other constituents in licorice root-their relative astringency and radical scavenging effects. Chem Pharm Bull. 1988;36:2090-2097.

32. Paranjpe $\mathrm{M}$ and Muller-Goymann CC. Nanoparticle-mediated pulmonary drug delivery: a review. Int J Mol Sci. 2014;15:5852-5873.

33. Kim TH, Jiang HH, Youn YS, Park CW, Tak KK, Lee S, Kim H, Jon S, Chen X, and Lee KC. Preparation and characterization of water-soluble albumin-bound curcumin nanoparticles with improved antitumor activity. Int J Pharm. 2011;403:285-291.

34. Wei Y, Li L, Xi Y, Qian S, Gao Y, and Zhang J. Sustained release and enhanced bioavailability of injectable scutellarin-loaded bovine serum albumin nanoparticles. Int J Pharm. 2014;476:142-148.

35. Valeur B. Molecular fluorescence principles and applications. Wiley-VCH: Weinheim; 2002; 73-124.

36. Yuan J-L, lv Z, Liu Z-G, Hu Z, and Zou G-L. Study on interaction between apigenin and human serum albumin by spectroscopy and molecular modeling. $J$ Photochem Photobiol A. 2007;191:104-113. 
702 37. Naveenraj S and Anandan S. Binding of serum albumins with bioactive substances $703 \quad$ Nanoparticles to drugs. J Photochem Photobiol C. 2013;14:53-71.

704 38. Tayeh N, Rungassamy $\mathrm{T}$, and Albani JR. Fluorescence spectral resolution of 705 tryptophan residues in bovine and human serum albumins. J Pharm Biomed Anal. $706 \quad 2009 ; 50: 107-116$.

707

708

709

710

711

712

713

714

715

716

717

718

719

720

721

722

723

724

725

39. Zhao X-N, Liu Y, Niu L-Y, and Zhao C-P. Spectroscopic studies on the interaction of bovine serum albumin with surfactants and apigenin. Spectrochim Acta A Mol Biomol Spectrosc. 2012;94:357-364.

40. Lin $\mathrm{C}-\mathrm{Z}, \mathrm{Hu} \mathrm{M}, \mathrm{Wu} \mathrm{A}-\mathrm{Z}$, and Zhu C-C. Investigation on the differences of four flavonoids with similar structure binding to human serum albumin. $J$ Pharm Anal. 2014;4:392-398.

41. Cao H, Liu Q, Shi J, Xiao J, and Xu M. Comparing the Affinities of Flavonoid Isomers with Protein by Fluorescence Spectroscopy. Anal Lett. 2008;41:521-532.

42. Shang $\mathrm{Y}$ and $\mathrm{Li} \mathrm{H}$. Studies of the interaction between apigenin and bovine serum albumin by spectroscopic methods. Russ J Gen Chem. 2010;80:1710-1717.

43. Tang L, Jia W, and Zhang D. The effects of experimental conditions of fluorescence quenching on the binding parameters of apigenin to bovine serum albumin by response surface methods. Luminescence. 2014;29:344-351.

44. Stahl K, Claesson M, Lilliehorn P, Linden H, and Backstrom K. The effect of process variables on the degradation and physical properties of spray dried insulin intended for inhalation. Int J Pharm. 2002;233:227-237.

45. Jensen DM, Cun D, Maltesen MJ, Frokjaer S, Nielsen HM, and Foged C. Spray drying of siRNA-containing PLGA nanoparticles intended for inhalation. J Control Release. 2010;142:138-145. 
726 46. Aquino RP, Prota L, Auriemma G, Santoro A, Mencherini T, Colombo G, and Russo

727 P. Dry powder inhalers of gentamicin and leucine: formulation parameters, aerosol 728 performance and in vitro toxicity on CuFil cells. Int J Pharm. 2012;426:100-107.

729 47. Yang X-F, Xu Y, Qu D-S, and Li H-Y. The influence of amino acids on aztreonam 730 spray-dried powders for inhalation. Asian J Pharm Sci. 2015;10:541-548.

731

732

733

734

735

736

737

738

739

740

741

742

743

744

745

746

747

748

749

750

48. Wu L, Miao X, Shan Z, Huang Y, Li L, Pan X, Yao Q, Li G, and Wu C. Studies on the spray dried lactose as carrier for dry powder inhalation. Asian J Pharm Sci. 2014;9:336-341.

49. Listiohadi Y, Hourigan J, Sleigh R, and Steele R. Thermal analysis of amorphous lactose and $\alpha$-lactose monohydrate. Dairy Sci Technol. 2009;89:43-67.

50. Němec I and Mička Z. FTIR and FT Raman study of L-leucine addition compound with nitric acid. J Mol Struct. 1999;482-483:23-28.

51. Newman AW and Byrn SR. Solid-state analysis of the active pharmaceutical ingredient in drug products. Drug Discov Today. 2003;8:898-905.

52. Sou T, Kaminskas LM, Nguyen T-H, Carlberg R, McIntosh MP, and Morton DAV. The effect of amino acid excipients on morphology and solid-state properties of multicomponent spray-dried formulations for pulmonary delivery of biomacromolecules. Eur J Pharm Biopharm. 2013;83:234-243.

53. Mangal S, Meiser F, Tan G, Gengenbach T, Denman J, Rowles MR, Larson I, and Morton DAV. Relationship between surface concentration of 1-leucine and bulk powder properties in spray dried formulations. Eur J Pharm Biopharm. 2015;94:160169.

54. Gombás Á, Szabó-Révész P, Kata M, Regdon G, Jr., and Erős I. Quantitative Determination of Crystallinity of $\alpha$-Lactose Monohydrate by DSC. $J$ Therm Anal Calorim. 2002;68:503-510. 
751 55. Raula J, Seppälä J, Malm J, Karppinen M, and Kauppinen EI. Structure and 752 Dissolution of 1-Leucine-Coated Salbutamol Sulphate Aerosol Particles. AAPS 753 PharmSciTech. 2012;13:707-712.

754 56. Yang MY, Chan JGY, and Chan H-K. Pulmonary drug delivery by powder aerosols. $J$ $755 \quad$ Control Release. 2014;193:228-240.

756

757

758

759

760

761

762

763

764

765

766

767

768

769

770

771

772

773

774

775

776

57. Chow AL, Tong HY, Chattopadhyay P, and Shekunov B. Particle Engineering for Pulmonary Drug Delivery. Pharm Res. 2007;24:411-437.

58. Liang Z, Ni R, Zhou J, and Mao S. Recent advances in controlled pulmonary drug delivery. Drug Discov To. 2015;20:380-389.

59. Heyder J, Gebhart J, Rudolf G, Schiller CF, and Stahlhofen W. Deposition of particles in the human respiratory tract in the size range 0.005-15 $\mu \mathrm{m}$. J Aerosol Sci. $1986 ; 17: 811-825$.

60. Raula J, Seppälä J, Malm J, Karppinen M, Kauppinen EI. Structure and dissolution of L-leucine-coated salbutamol sulphate aerosol particles. AAPS PharmSciTech. 2012; 13:707-12.

61. May S, Jensen B, Wolkenhauer M, Schneider M, Lehr CM. Dissolution techniques for in vitro testing of dry powders for inhalation. Pharm Res. 2012;29:2157-66.

62. Knieke C, Azad MA, To D, Bilgili E, Davé RN. Sub-100 micron fast dissolving nanocomposite drug powders. Powder Technol. 2015;271:49-60.

63. Patel B, Gupta N, and Ahsan F. Particle engineering to enhance or lessen particle uptake by alveolar macrophages and to influence the therapeutic outcome. Eur $J$ Pharm Biopharm. 2015;89:163-174.

64. Kou X, Chan LW, Steckel H, and Heng PWS. Physico-chemical aspects of lactose for inhalation. Adv Drug Deliv Rev. 2012;64:220-232.

65. Pilcer G, Wauthoz N, and Amighi K. Lactose characteristics and the generation of the aerosol. Adv Drug Deliv Rev. 2012;64:233-256. 
777 66. Prota L, Santoro A, Bifulco M, Aquino RP, Mencherini T, and Russo P. Leucine 778 enhances aerosol performance of Naringin dry powder and its activity on cystic 779 fibrosis airway epithelial cells. Int J Pharm. 2011;412:8-19.

780 67. Taki M, Marriott C, Zeng X-M, and Martin GP. Aerodynamic deposition of 781 combination dry powder inhaler formulations in vitro: A comparison of three 782 impactors. Int J Pharm. 2010;388:40-51.

783 68. Davis SS. Delivery of peptide and non-peptide drugs through the respiratory tract. 784 Pharm Sci Techn To. 1999;2:450-456.

69. Musante CJ, Schroeter JD, Rosati JA, Crowder TM, Hickey AJ, and Martonen TB. 786

787

788

71. Chew NY and Chan HK. The role of particle properties in pharmaceutical powder inhalation formulations. $J$ Aerosol Med. 2002;15:325-330.

72. Brand-Williams W, Cuvelier ME, and Berset C. Use of a free radical method to evaluate antioxidant activity. LWT - Food Science and Technology. 1995;28:25-30.

73. Villaño D, Fernández-Pachón MS, Moyá ML, Troncoso AM, and García-Parrilla MC. Radical scavenging ability of polyphenolic compounds towards $\mathrm{DPPH}^{\bullet}$ free radical. Talanta. 2007;71:230-235.

74. Al Shaal L, Shegokar R, and Muller RH. Production and characterization of antioxidant apigenin nanocrystals as a novel UV skin protective formulation. Int $J$ Pharm. 2011;420:133-140. 
801 75. Papay Z.E AI. Study on the antioxidant activity during the formulation of biological 802 active ingredient. European Scientific Journal. 2014;Special Edition 3:252-257.

803 76. Yang J, Guo J, and Yuan J. In vitro antioxidant properties of rutin. LWT - Food $804 \quad$ Science and Technology. 2008;41:1060-1066.

805 77. Natella F, Nardini M, Di Felice M, and Scaccini C. Benzoic and Cinnamic Acid 806 Derivatives as Antioxidants: Structure-Activity Relation. J Agr Food Chem. $807 \quad 1999 ; 47: 1453-1459$.

808 78. Roche M, Rondeau P, Singh NR, Tarnus E, and Bourdon E. The antioxidant properties $809 \quad$ of serum albumin. FEBS Lett. 2008;582:1783-1787.

810 79. Fang R, Jing H, Chai Z, Zhao G, Stoll S, Ren F, Liu F, and Leng X. Study of the 811 physicochemical properties of the BSA: flavonoid nanoparticle. Eur Food Res $812 \quad$ Technol. 2011;233:275-283.

813

814 
815 TABLES

816 Table 1

817 Aerosol particle sizes of spray-dried nanoparticles with Sympatec HELOS laser 818 diffractometer in $\mu \mathrm{m}$.

\begin{tabular}{llll}
\hline & Excipient-free & Lactose & L-leucine \\
\cline { 2 - 4 } ED (\%) & $91.862 \pm 2.735$ & $93.950 \pm 1.046$ & $95.183 \pm 0.667$ \\
FPF (\%) & $65.617 \pm 3.422$ & $58.463 \pm 6.031$ & $66.090 \pm 2.777$ \\
MMAD ( $\boldsymbol{\mu m})$ & $3.210 \pm 0.069$ & $3.130 \pm 0.001$ & $2.123 \pm 0.098$ \\
GSD ( $\boldsymbol{\mu m})$ & $2.823 \pm 0.113$ & $2.270 \pm 0.212$ & $1.887 \pm 0.063$ \\
RD (\%) & $99.1 \pm 5.012$ & $94.7 \pm 4.091$ & $96.3 \pm 2.161$ \\
\hline
\end{tabular}

Table 2

822 Aerodynamic characteristic of spray-dried nanoparticles.

\begin{tabular}{llll}
\hline & Excipient-free & Lactose & L-leucine \\
\cline { 2 - 4 } $\mathbf{D}_{\mathbf{1 0}}$ & $1.033 \pm 0.032$ & $1.020 \pm 0.070$ & $0.843 \pm 0.680$ \\
$\mathbf{D}_{\mathbf{5 0}}$ & $3.030 \pm 0.092$ & $3.107 \pm 0.102$ & $2.473 \pm 0.300$ \\
$\mathbf{D}_{\mathbf{9 0}}$ & $7.110 \pm 0.306$ & $7.117 \pm 0.337$ & $5.287 \pm 0.670$ \\
\hline
\end{tabular}




\section{FIGURE CAPTIONS}

826 Figure 1. Molecular structure of apigenin.

827 Figure 2. Fluorescence emission spectra of BSA solution, BSA nanoparticles and BSA-Api 828 nanoparticles. The excitation wavelength was set to $285 \mathrm{~nm}$ (EM: emission, EXC: $829 \quad$ excitation)

830 Figure 3. Three dimensional fluorescence emission maps and two dimensional contour maps of empty BSA nanoparticles and BSA-Api nanoparticles. Color scale displays the range of observed fluorescence intensities.

Figure 4. A) FT-IR spectra of apigenin (1), BSA (2) and the excipient-free spray-dried BSA-Api nanoparticles (3).

Figure 5. XRPD diffraction pattern of raw apigenin and the formulations.

Figure 6. A) DSC thermograms of apigenin (1), BSA (2) physical mixture (3) and the excipient-free spray dried BSA-Api nanoparticles (4).

B) DSC spectra of apigenin (1), BSA (2), physical mixture (3) and the spray-dried BSA-Api nanoparticles with lactose (4).

C) DSC spectra of apigenin (1), BSA (2), physical mixture (3) and the spray-dried BSA-Api nanoparticles with L-leucine (4).

Figure 7. A) Solubility of spray dried BSA-Api formulations in PBS buffer and modified simulated lung fluid (mSLF).

B) Dissolution of apigenin from the formulations as a function of time in modified simulated lung fluid (mSLF). 
Figure 8. NGI deposition pattern of the spray dried BSA-Api formulations.

Figure 9. SEM images of raw apigenin (1), excipient-free spray dried BSA-Api nanoparticles (2), spray-dried BSA-Api nanoparticles with lactose (3), spray-dried BSA-Api nanoparticles with L-leucine (4) 20000 x magnification.

Department of Pharmaceutics

861 Semmelweis University

862 Hőgyes E. Street 7-9,

863 H-1092 Budapest, Hungary

864 Tel/Fax: +3612017-0914; Tel.: +361476-3600/53066, 53087 BSA-Apigenin nanoparticles (NP) and spray-dried nanoparticles (SD) with excipients. The antioxidant activity is expressed as the inhibition of $\mathrm{DPPH}^{\bullet}$ free radical in percent.

To whom correspondence should be addressed:

antal.istvan@pharma.semmelweis-univ.hu 
1 Study on the pulmonary delivery system of apigenin loaded albumin nanocarriers with antioxidant activity

3 Zsófia Edit Pápay ${ }^{1}$, Annamária Kósa, $\mathrm{PhD}^{2}$, Béla Böddi, $\mathrm{PhD}^{2}$, Zahra Merchant ${ }^{3}$, Imran Y

4 Saleem, $\mathrm{PhD}^{4}$, Mohammed Gulrez Zariwala, $\mathrm{PhD}^{5}$, Imre Klebovich, $\mathrm{PhD}{ }^{1}$, Satyanarayana

5 Somavarapu, $\mathrm{PhD}^{3 *}$, István Antal, $\mathrm{PhD}^{1^{*}}$

$6{ }^{1}$ Department of Pharmaceutics, Semmelweis University, Hőgyes E. Street 7-9, H-1092

7 Budapest, Hungary

$8{ }^{2}$ Department of Plant Anatomy, Institute of Biology, Eötvös Lóránd University, Pázmány

9 Péter Street 1/C, Budapest, Hungary

$10{ }^{3}$ Department of Pharmaceutics, UCL School of Pharmacy, 29-39 Brunswick Square, London 11 WC1N 1AX, United Kingdom

$12{ }^{4}$ Formulation and Drug Delivery Research, School of Pharmacy and Biomolecular Sciences, 13 Liverpool John Moores University, Liverpool, United Kingdom

$14{ }^{5}$ Department of Biomedical Science, Faculty of Science and Technology, University of 15 Westminster, 115 New Cavendish Street, London, W1W 6UW, United Kingdom

$16 *$ To whom correspondence should be addressed:

17 antal.istvan@pharma.semmelweis-univ.hu

18 Department of Pharmaceutics

19 Semmelweis University

20 Hőgyes E. Street 7-9,

21 H-1092 Budapest, Hungary

22 Tel/Fax: +3612017-0914; Tel.: +361476-3600/53066, 53087

23 Running title:

24 Albumin-Apigenin Nanoparticles against Lung Injury 25 
Background: Respiratory diseases are mainly derived from acute and chronic inflammation of the alveoli and bronchi. The pathophysiological mechanisms of pulmonary inflammation mainly arise from oxidative damage that could ultimately lead to acute lung injury (ALI).

\section{ABSTRACT} Apigenin (Api) is a natural polyphenol with prominent antioxidant and anti-inflammatory properties in the lung. Inhalable formulations consist of nanoparticles (NPs) have several advantages over other administration routes therefore this study investigated the application of apigenin loaded bovine serum albumin nanoparticles (BSA-Api-NPs) for pulmonary delivery.

Methods: Dry powder formulations of BSA-Api-NPs were prepared by spray drying and characterized by laser diffraction particle sizing, scanning electron microscopy, differential scanning calorimetry and powder X-ray diffraction. The influence of dispersibility enhancers (lactose monohydrate and L-leucine) on the in vitro aerosol deposition using a next generation impactor (NGI) was investigated in comparison to excipient-free formulation. The dissolution of Api was determined in simulated lung fluid by using Franz cell apparatus. The antioxidant activity was determined by 2,2-Diphenyl-1-picrylhydrazyl (DPPH*) free radical scavenging assay.

Results: The encapsulation efficiency and the drug loading was measured to be $82.61 \pm$ $4.56 \%$ and $7.51 \pm 0.415 \%$. The optimized spray drying conditions were suitable to produce particles with low residual moisture content. The spray dried BSA-Api-NPs possessed good the aerodynamic properties due to small and wrinkled particles with low mass median aerodynamic diameter, high emitted dose and fine particle fraction. The aerodynamic properties was enhanced by leucine and decreased by lactose, however, the dissolution was reversely affected. The $\mathrm{DPPH}^{*}$ assay confirmed that the antioxidant activity of encapsulated Api was preserved. 
49 Conclusion: This study provides evidence to support that albumin nanoparticles are suitable

50 carriers of Api and the use of traditional or novel excipients should be taken into

51 consideration. The developed BSA-Api-NPs is a novel delivery system against lung injury

52 with potential antioxidant activity.

53 Keywords: aerosol distribution, inhaled therapy, modeling

54 


\section{INTRODUCTION}

Respiratory diseases are thought to be mainly derived from acute and chronic inflammation of the alveoli and the bronchi. The pathophysiological mechanisms of pulmonary inflammation arise from several factors, including oxidative damage due to cytotoxic mediators that may ultimately lead to acute lung injury (ALI), acute respiratory distress syndrome (ARDS) and cancer ${ }^{1}$. A growing body of scientific data suggests that natural occurring compounds possess preventive and therapeutic properties with inherent low toxicity $^{2}$. Among phytochemicals, apigenin (Api, Figure 1) is a promising candidate as a therapeutic agent, mainly due to its antioxidant and anti-inflammatory properties ${ }^{3-6}$. It has been demonstrated that Api has protective effects against bleomycin-induced lung fibrosis in rats which is associated with its antioxidant and anti-inflammatory capacities ${ }^{7}$. Another study provided evidence that Api has was able to decrease oxidative stress and inflammation on paraquat-induced ALI in mice ${ }^{8}$ and reduced the pathological alterations of pulmonary tissue in acute pancreatitis associated ALI, therefore suggesting protection in the lung 9 . Furthermore, Api has anti-inflammatory effect owing to significant inhibition of proinflammatory cytokines, activator protein (AP-1) and cyclooxygenase-2 (COX-2) in human pulmonary epithelial cells ${ }^{10}$ and in mice as well ${ }^{11}$. However, Api's has low water solubility $(2.16 \mu \mathrm{g} / \mathrm{ml}$ at $\mathrm{pH}$ 7.5) and therefore it was recently classified as BCS (Biopharmaceutical Classification System) II. drug ${ }^{12}$.

Encapsulation and delivery of phytoconstituents with health effects has attracted much attention in recent years. Developing a suitable carrier system is essential to improve the overall activity and reduce the possible toxicity of these agents ${ }^{13}$. Among the potential carrier systems, serum albumin nanoparticles have notable advantages including biodegradability, non-antigenicity and cell-targeting ability ${ }^{14,15}$. Moreover, albumin provide exceptional ligand binding capacity for various drugs owing to three homologous domains with two separate 
80 helical subdomains ${ }^{16}$. Studies reported the successful incorporation of flavonoids into albumin 81 nanoparticles that can improve their stability ${ }^{17}$ and antitumor activity ${ }^{18}$.

82 Pulmonary delivery of pharmacologically active ingredients are extensively studied due to prominent advantages over other delivery routes of administration ${ }^{19}$. The lungs have a large

84 surface area, limited enzymatic activity and high permeability therefore drugs can be delivered either locally for the treatment of respiratory diseases or systematically in order to e.g. avoid first pass metabolism ${ }^{20}$. Dry Powder Inhaler (DPI) products offer precise and reproducible delivery of fine drug particle fraction to the deep lung and recent studies have proved that these are more cost effective than other products ${ }^{21}$. This non-invasive delivery route could be suitable for poorly water soluble drugs in nanoparticles with increased solubility $^{22}$. It is also well recognized that nanoparticles have benefits over other carriers in the micron scale such as controlled drug release, avoiding mucociliary clearance and improve deposition $^{23,24}$. Albumin is naturally present in the body, as well as in the lung epithelium ${ }^{24}$, moreover, the body can absorb proteins into the bloodstream by transcytosis which occurs deep in the lung and allows drug molecules to pass through cell membrane ${ }^{25}$. Therefore the presence of BSA in the nanoparticle system increases membrane permeability, may facilitate epithelial cell uptake and translocation through the alveo-capillary barrier of the lung ${ }^{26}$. It was proved that albumin nanoparticles have high biocompatibility in a wide dose range and remained longer in the lungs with low systemic exposure ${ }^{24}$. Thus encapsulation of apigenin into albumin nanoparticles would enhance its solubility and distribution in the lung. However, the formulation of dry powders with optimal aerodynamic properties for pulmonary drug delivery is challenging. Spray drying is a technique for manufacturing respirable dry powders in one step. During the process, the liquid phase is atomized into droplets that dry rapidly in the drying chamber due to the compressed air. The process conditions like heat, flow rate, aspiration rate and pump rate also determine the quality of the product. The thermal 
105 degradation caused by overheating can be avoided by the rapid evaporation of the solvent ${ }^{27}$.

106 Hence, it is suitable for drying colloidal systems resulting in uniform particle morphology.

107 Nanoparticle delivery systems targeted to the lungs offer several advantages such as sustained 108 release, increased local drug concentration and targeted site of action ${ }^{28}$. Moreover, improved 109 drug solubility, uniform dose distribution and fewer side effects can be achieved, compared to 110 conventional dry powders. In general, respirable nanoparticles are embedded in microparticles 111 in aerodynamic size range ${ }^{26}$.

112 The aim of this work was to develop a novel dry powder formulation against ALI 113 caused by oxidative stress. The prepared albumin nanoparticles were characterized in terms of 114 size, zeta potential and drug loading, additionally the fluorescence properties were 115 investigated. Following this, the nanoparticles were spray dried with two types of excipients, 116 namely a traditional lactose monohydrate and a novel amino acid, L-leucine. In vitro aerosol 117 deposition patterns were determined in comparison to excipient-free formulation using a next 118 generation impactor (NGI) and dissolution test was performed in simulated lung fluid by 119 using Franz cell apparatus. Laser diffraction particle sizing, morphology and residual moisture 120 content were measured along with the antioxidant activity.

\section{MATERIALS AND METHODS}

\section{MATERIALS}

Apigenin (Api) was purchased from (purity > 99\%) Hangzhou Dadyangchem Co., Ltd. (China). Bovine serum albumin powder (BSA, purity $\geq 98 \%$ ), L-leucine, analytical grade chloroform, acetonitrile and trifluoroacetic acid (TFA) were obtained from Sigma Aldrich Ltd. (Dorset, UK). Lactohale ${ }^{\circledR}$ LH 230 was supplied by Friesland Foods Domo (Amersfoort, The Netherlands). 2,2-Diphenyl-1-picrylhydrazyl (DPPH*) free radical was purchased from Sigma-Aldrich (Darmstadt, Germany). For the solubility and drug release study, PBS buffer was purchased (Sigma Aldrich Ltd., Dorset, UK) and simulated lung fluid modified with 
$130 \quad 0.02 \%(\mathrm{w} / \mathrm{v})(\mathrm{mSLF})$ was prepared. All of the materials for the mSLF were purchased from

131 Sigma Aldrich Ltd. (Dorset, UK).

\section{METHODS}

133 2.1. Preparation of BSA-NPs

134 BSA nanoparticles were prepared using a nanoparticle albumin bound technology with 135 minor modifications ${ }^{29}$. Briefly, $1000 \mathrm{mg}$ of BSA was dissolved in $50 \mathrm{ml}$ of distilled water 136 saturated with chloroform. Separately, $100 \mathrm{mg}$ of Api was dissolved in $3 \mathrm{ml}$ of chloroform 137 saturated with water and ultrasonicated for 10 minutes. These two solutions were mixed and 138 ultrasonicated for 20 minutes with a probe-type sonicator (MSE Soniprep 150 Ultrasonic 139 Processor, MSE Ltd., London, UK) on ice. After homogenization, the chloroform was 140 evaporated by rotary evaporator (Rotavapor ${ }^{\circledR}$ R-10, BÜCHI Labortechnik AG, Flawil, 141 Switzerland) at $25^{\circ} \mathrm{C}$ for 15 minutes. The obtained nanoparticles were filtered through filter 142 paper $(0.45 \mu \mathrm{m}$, Ficher Scientific Ltd., Loughborough, UK) and further spray dried.

\section{2.2. Characterization of BSA-Api-NPs}

\section{$144 \quad$ 2.2.1. Particle size and zeta potential analysis}

The average particle size and polydispersity index (PDI) were determined by dynamic

146 light scattering (DLS) using Zetasizer Nano ZS instrument (Malvern Instruments Ltd., 147 Worcestershire, UK). Zeta potential of the particles was quantified with laser doppler 148 velocimetry (LDV) using the same instrument. All measurements were performed in triplicate $149(\mathrm{n}=3)$ at $25^{\circ} \mathrm{C}$ and presented as mean \pm standard deviation (SD).

\subsubsection{Determination of drug loading and encapsulation efficiency}

To determine the amount of Api, $1 \mathrm{ml}$ sample from the BSA-Api formulation was

152 withdrawn and the apigenin content was determined in $\mathrm{mg} / \mathrm{ml}$ by adding $5 \mathrm{ml}$ of dimethyl 153 sulfoxide and methanol (DMSO:MeOH, 50:50\% v/v) and sonicated for 10 minutes. The exact 154 concentrations were determined after filtration $(0.22 \mu \mathrm{m}$ ) by HPLC 1260 (Agilent 
155 Technologies Inc., Santa Clara, USA) using reverse-phase $\mathrm{C}_{18}$ column (Phenomenex ${ }^{\circledR}$, $156250 \times 4.6 \mathrm{~mm}, 4 \mu \mathrm{m})$ as the stationary phase. The temperature was set to $25^{\circ} \mathrm{C}$. The mobile 157 phase consisted of $40 \%$ acetonitrile and $60 \%$ water containing $0.1 \%(\mathrm{v} / \mathrm{v})$ TFA. The system 158 was run isocratically at the flow rate of $1.2 \mathrm{ml} / \mathrm{min}$ and the Api was detected at $340 \mathrm{~nm}\left(\mathrm{t}_{\mathrm{R}}=\right.$ 159 8.3). The injection volume was set to $10 \mu$ l. A calibration curve was conducted by diluting 160 stock solution $(0.1 \mathrm{mg} / \mathrm{ml})$ with $\mathrm{R}^{2}$ value of 0.999 .

161 The drug loading efficiency (DL, \%) and encapsulation efficiency (EE, \%) were 162 calculated according to the equations (Eq. 1. and 2.), comparing the encapsulated Api content $163\left(\mathrm{mg} / \mathrm{ml}, \mathrm{W}_{\text {encapsulated }}\right)$ to total nanoparticle system which means the weighted amount of BSA 164 and Api together $\left(\mathrm{mg} / \mathrm{ml}, \mathrm{W}_{\text {total }}\right)$ and the amount of Api $\left(\mathrm{mg} / \mathrm{ml}, \mathrm{W}_{\text {theoretical }}\right)$ used in the 165 formulations.

\subsubsection{Fluorescence spectroscopy}

$$
\begin{gathered}
D L(\%)=\frac{W_{\text {encapsulated }}}{W_{\text {total }}} \times 100 \\
E E(\%)=\frac{W_{\text {encapsulated }}}{W_{\text {theoretical }}} \times 100
\end{gathered}
$$

The fluorescence emission spectra of BSA and BSA-Api-NPs were measured with Jobin Yvon-Horiba Fluoromax-3 (Paris, France) spectrofluorometer. The samples which contained the nanoparticles were diluted 10 times and the fluorescence emission spectra were recorded between 300 and $450 \mathrm{~nm}$ at $25{ }^{\circ} \mathrm{C}$ where the excitation wavelength was set to 285 $\mathrm{nm}$. The data collection frequency was $0.5 \mathrm{~nm}$ and the integration time was $0.2 \mathrm{~s}$. The excitation slit was set at a bandpass width of $2 \mathrm{~nm}$ and the emission slit at $5 \mathrm{~nm}$. Each spectrum was recorded three times and the mean values were calculated automatically. The SPSERV V3.14 software (C) Csaba Bagyinka, Institute of Biophysics, Biological Research Center of the Hungarian Academy of Sciences, Szeged, Hungary) was used for baseline correction, for five point linear smoothing and for the correction to the wavelength-dependent 
179 sensitivity changes of the spectrofluorometer. The subtraction of the Raman band at $390 \mathrm{~nm}$ 180 was performed.

181 To obtain the three-dimensional projections and contour maps of fluorescence spectra 182 of the samples, the fluorescence emission were recorded from 265 to $450 \mathrm{~nm}$ using different 183 excitation wavelengths from 250 to $310 \mathrm{~nm}$ with $10 \mathrm{~nm}$-steps with the same instrument 184 mentioned above. All emission scan ranges were set to start at least $15 \mathrm{~nm}$ away from the 185 corresponding excitation wavelengths. Other settings were similar as described above. Each 186 spectrum was recorded three times and the mean values were calculated automatically. The 187 three-dimensional fluorescence spectra were visualized with the software SURFER Version 18810 (Golden Software, Inc., Colorado, USA). Spectra were combined together into a three 189 dimensional surface data set with axes of excitation and emission wavelengths and 190 fluorescence intensity. Data were also converted into two dimensional contour maps.

\section{2.3. Spray drying of BSA-Api-NPs}

192 Spray drying of the BSA-Api formulations without excipient and in the presence of 193 lactose monohydrate $(50 \%, \mathrm{w} / \mathrm{w})$ and L-leucine $(9 \%, \mathrm{w} / \mathrm{w})$ were carried out in a Büchi 290 194 Mini Spray Dryer (BÜCHI Labortechnik AG, Flawil, Switzerland). The concentration of the 195 excipients were with respect to the mass of the nanoparticles before spray drying. The 196 following operating conditions were used based on pilot experiments: inlet temperature 120 $197{ }^{\circ} \mathrm{C}$, approximate outlet temperature $65-70{ }^{\circ} \mathrm{C}$, the drying airflow $600 \mathrm{~L} / \mathrm{h}$, aspiration rate $198100 \%\left(35 \mathrm{~m}^{3} / \mathrm{h}\right)$, the nozzle diameter was a $0.1 \mathrm{~mm}$ and the liquid feed rate was set to 5 $199 \mathrm{ml} / \mathrm{min}$. Each preparation were carried out in triplicate. Following spray drying, the powders 200 were collected from the lower part of the cyclone and the collecting vessel, stored in tightly 201 sealed glass vials under vacuum at room temperature.

202 2.4. Characterization of spray-dried BSA-Api-NPs

203

\subsubsection{Determination of residual moisture}


The moisture content of the spray-dried powders was measured by using Karl Fischer titration (Metrohm 758 KFD Titirino, Metrohm AG, Lichtenstein, Switzerland). For that purpose approx. $100 \mathrm{mg}$ of the product was analysed and the instrument was previously calibrated with $10 \mu$ distilled water. The evaluation was conducted in triplicate and the standard deviation calculated.

\subsubsection{Fourier-Transform Infrared Spectroscopy (FT-IR)}

FT-IR spectra of BSA-Api spray-dried samples were evaluated using a PerkinElmer

Spectrum 100 FT-IR spectrometer equipped with Universal ATR (Attenuated Total

Reflectance) accessory (PerkinElmer Inc., Waltham, USA). Approximately $2 \mathrm{mg}$ of the solid

samples were placed between the plate and the probe. The spectra were recorded with 3 scans,

214 in the frequency range between $4000-600 \mathrm{~cm}^{-1}$ and with a resolution of $4 \mathrm{~cm}^{-1}$ at room

\subsubsection{X-ray powder diffraction (XRPD)}

XRPD diffractograms were obtained using an X-ray diffractometer (MiniFlex600 Rigaku Corporation, Tokyo, Japan). The analyses were performed at room temperature and the samples were scanned from $2^{\circ}$ to $40^{\circ} 2 \theta$ using a scanning speed of $2 \%$ min with a step size of $0.05^{\circ}$.

\subsubsection{Differential scanning calorimetry (DSC) analysis}

The spray-dried formulations were characterized by DSC (DSC Q2000 module; TA Instruments, New Castle, UK) which was calibrated using indium. Samples (3-5 mg) were weighed accurately and analyzed in sealed and pierced aluminium hermetic pans (TA Instruments). The pans were equilibrated at $25^{\circ} \mathrm{C}$ and then heated at a rate of $10{ }^{\circ} \mathrm{C} / \mathrm{min}$ in a range of $50-400{ }^{\circ} \mathrm{C}$.

\subsubsection{Aerosol particle size analysis and redispersibility in water}



diffractometer (Sympatec GmbH System-Partikel-Technik, Clausthal-Zellerfeld, Germany). The powders were dispersed by compressed air ( $4-5$ bar) into the measuring zone of the laser beam. The optical lens $(0.45-87.5 \mu \mathrm{m}$ size range $)$ focused onto the detector to collect the diffracted light for calculation of size distribution. The values of 10 th $\left(\mathrm{D}_{10}\right), 50$ th $\left(\mathrm{D}_{50}\right)$ and 90th $\left(\mathrm{D}_{90}\right)$ of the cumulative particle size distribution are generated. Samples were measured 234 in triplicate.

235 The particle size was also determined after spray drying. $5 \mathrm{mg}$ dry powder of each 236 formulation could be easily redispersed in $5 \mathrm{ml}$ distilled water and the particle size was 237 determined without any further dilution by the above mentioned Zetasizer Nano ZS 238 instrument (Malvern Instruments Ltd., Worcestershire, UK).

\subsubsection{Solubility and Drug release studies of BSA-Api formulations}

The solubility of BSA-Api formulations were determined in PBS buffer (pH 7.4) and

241 in modified simulated lung fluid (mSLF, $\mathrm{pH}$ 7.4) which contained $0.02 \%(\mathrm{w} / \mathrm{v})$ DPPC was

242 prepared according to Son and McConville ${ }^{30} .50 \mathrm{mg}$ of samples of spray dried powders were 243 added to $100 \mathrm{~mL}$ solvent and shaken $(150 \mathrm{rpm})$ for $2 \mathrm{~h}$ at $37^{\circ} \mathrm{C}$. At predetermined time points

$2441 \mathrm{~mL}$ of samples were taken from each dissolution media and replaced with the same volume

245 of fresh medium. All of the samples were diluted with $1 \mathrm{ml}$ methanol and filtered with 246 Amicon ${ }^{\circledR}$ Ultra Centrifugal filters (30K, Merck Millipore, Merck KGaA, Germany) prior to 247 the injection and the amount of apigenin was determined by HPLC-UV method.

248 The in vitro drug release study of the three formulations were conducted with Franz cell 249 apparatus. The mSLF was used as dissolution media and $0.45 \mu \mathrm{m}$ cellulose acetate membrane 250 filter (Sartorius AG, Goettingen, Germany) was applied. Briefly, an accurately weighed 251 amount $(10 \mathrm{mg})$ of spray dried nanoparticles of each formulations were scattered onto the 252 membrane which was previously wetted with the dissolution media for 1 hour. $1 \mathrm{ml}$ of 
253 samples were withdrawn at various time intervals for 5 hours and replaced with fresh 254 dissolution medium. After the measurement, membrane was rinsed with $2 \mathrm{ml}$ of $\mathrm{MeOH}$ and 255 the drug content of the possibly remained powders was determined. The sample preparation 256 and the measurement was the same as mentioned above. The cumulative amount of apigenin 257 release over the time was plotted for each formulations. All measurements were performed in 258 triplicate.

\section{2.4.7. Aerosol delivery of BSA-Api formulations}

260 In vitro aerodynamic performance of BSA-Api formulations was assessed using the 261 next generation impactor (NGI; Copley Scientific Ltd., Nottingham, UK), connected 262 sequentially to a low capacity pump via the critical flow controller (Model LCP5; Copley 263 Scientific Ltd., Nottingham, UK). During the measurement the pump was operated at air flow 264 rate of $60 \mathrm{~L} / \mathrm{min}$ for $4 \mathrm{~s}$. The $3 \times 10 \mathrm{mg}$ powder aliquots from each formulation were loaded 265 manually into gelatine capsules (size 3) and placed into the inhaler device (Cyclohaler ${ }^{\circledR}$, 266 Pharmachemie, London, UK) which was connected to the NGI via an airtight rubber adaptor 267 and a stainless steel USP throat. The NGI stages were assembled with an induction port, a 268 pre-separator and a filter was placed in the final stage. Prior to the impaction, the collection 269 plates were uniformly coated with $1 \mathrm{ml}$ of $1 \%$ silicone oil in $\mathrm{N}$-hexane solution and allowed 270 to dry leaving a thin film of silicone oil on the plate surface in order to prevent the re271 entrainment of the particles and the pre-separator was filled with $15 \mathrm{ml}$ DMSO:MeOH $272(50: 50 \%, v / v)$ mixture. After the deposition of the powders in the NGI, the amount of each 273 formulation was cumulatively collected onto silicone-coated plates for each of the stages. The 274 inhaler, mouth piece, induction port, pre-separator and the collection plates were rinsed with 275 DMSO:MeOH (50:50\%, v/v) mixture, collected in volumetric flasks (10 or $25 \mathrm{ml})$ and made 276 up to volume. The samples were determined by using HPLC method as described previously. 277 To characterize the aerosol performance the following parameters were calculated based on 
278 the drug mass of each fraction: emitted dose (ED, \%): the percentage of the entire dose

279 depositing from the mouthpiece of the inhaler device and recovered dose (RD, \%): the total 280 recovered drug mass. The fine particle fraction $(\mathrm{FPF},<4.46 \mu \mathrm{m})$ is defined as the percentage 281 of the emitted dose which deposited from the Stage 2-7 and the micro orifice-collector 282 (MOC). The mass median aerodynamic diameter (MMAD) and geometric standard deviation 283 (GSD) were calculated from the inverse of the standard normal cumulative mass distribution 284 against the natural logarithm of the effective cut-off diameter of the respective stages. All 285 measurements were carried out in triplicate.

286 2.4.8. Particle morphology

287 Morphology of Api powder and spray-died nanoparticles was examined using 288 scanning electron microscopy (SEM) analysis. The dry powder of the formulations was 289 placed on the sample holder using double adhesive tape and gold coating ( $20 \mathrm{~nm}$ thickness) 290 was applied. Examinations were performed by FEI Inspect ${ }^{\mathrm{TM}} \mathrm{S} 50$ (Hillsboro, Oregon, USA) 291 scanning electron microscope at $20.00 \mathrm{kV}$ accelerating voltage. Original magnifications were $2928000 x, 10,000 x$ and $20,000 x$ with accuracy of $\pm 2 \%$.

$293 \quad$ 2.5. Antioxidant activity

294 The antioxidant activities of the prepared spray-dried formulations were compared to 295 the pure Api in order to investigate the effectiveness of the formulation. The free radical 296 scavenging activity was measured by using $\mathrm{DPPH}^{\bullet}$ method as described previously ${ }^{31}$ with 297 slight modifications. Methanolic stock solution of $0.1 \mathrm{mM} \mathrm{DPPH}$ reagent was freshly 298 prepared and protected from light. Standard curve was plotted between the DPPH 299 concentration (0.01-0.1 $\mathrm{mM}$ ) and absorbance, the linear relationship was calculated 300 graphically. $1 \mathrm{ml}$ of $\mathrm{MeOH}$ was added to the BSA-Api-NPs and the concentration of Api were 301 the same in each sample for the comparability. Thereafter $2 \mathrm{ml}$ of $0.06 \mathrm{mM} \mathrm{DPPH}^{\bullet}$ reagent 302 was added to the samples, vortex mixed for 10 seconds and protected from light. The 
absorbance at $517 \mathrm{~nm}$ was determined with spectrophotometer (UV-Vis spectrophotometer, Metertech SP-8001, Metertech Inc., Taipei, Taiwan) in every 15 minutes until the steady state (when no further discoloration could be observed). The addition of samples resulted decrease in the absorbance of $\mathrm{DPPH}^{\cdot}$ due to the scavenging activity of Api. The exact concentration of the free radical was calculated using the standard curve. To calculate the inhibition of the free radical DPPH${ }^{\bullet}$ the following equation (Eq.3.) was used:

$$
I(\%)=\frac{A_{0}-A_{s}}{A_{0}} \times 100
$$

Where I $(\%)$ is the inhibition in percent, $\mathrm{A}_{0}$ is the absorbance of the $\mathrm{DPPH}^{\bullet}$ solution and $\mathrm{A}_{\mathrm{s}}$ is the absorbance of the sample. All measurements were carried out triplicate and the data were expressed as the mean value $\pm \mathrm{SD}$.

\section{RESULTS AND DISCUSSION}

\subsection{Characterization of BSA-Api-NPs}

\subsubsection{Size, zeta potential and drug content}

Albumin is a natural protein that has been widely used as a macromolecular carrier for many drugs with low water solubility. Several techniques are available to prepare albumin nanoparticles including desolvation (coacervation), nab (nanoparticle albumin bound)technology and self-assembly ${ }^{14}$. In this study the BSA-Api-NPs were prepared by using modified nab-technology with ultrasonication. The achieved mean particle size of three samples was $376 \pm 7.824 \mathrm{~nm}$ with a polydispersity index of $0.285 \pm 0.01$. The size of albumin NPs less than $500 \mathrm{~nm}$ could localize effectively in the lung. The PDI value indicated narrow particle size distribution and the uniformity of the nanoparticles. The zeta potential was -19.20 $\pm 0.818 \mathrm{mV}$. The higher the zeta potential, the more stable the formulation is, less aggregation occurs $^{32}$. The EE was determined to be $82.61 \pm 4.56 \%$ and the DL was $7.51 \pm 0.415 \%$. Therefore these results confirmed the high encapsulation efficiency of apigenin by BSA-NPs and it can be attractive tool in encapsulation flavonoids for delivery. Similar data were found 
328 in the literature when encapsulating flavonoids into albumin nanoparticles. Human serum

329 albumin-bound curcumin nanoparticles resulted $7.2 \pm 2.5 \%$ loading efficiency ${ }^{33}$ and

330 scutellarin-loaded bovine serum albumin nanoparticles possess $64.46 \% \mathrm{EE}$ and $6.73 \% \mathrm{DL}^{34}$.

\section{$331 \quad 3.1 .2$. Fluorescence spectroscopy}

332 The phenomenon of fluorescence quenching can result from various inter and 333 intramolecular interactions such as energy transfer, conformational changes, complex 334 formation (static quenching) or collisional interaction (dynamic quenching). During static 335 quenching the quencher forms a stable non-fluorescent complex with the fluorophore, 336 however, during dynamic quenching it collides with the fluorophore and facilitates non337 radiative transitions to the ground $\operatorname{state}^{35}$. Therefore quenching of the intrinsic fluorescence of 338 the two tryptophan residues (Trp-134 and Trp-212) of BSA can offer information about the 339 changes in molecular microenvironment of these fluorophores, located in domain I and II, 340 respectively. Trp-134 residue is located close to the protein surface in a hydrophilic 341 environment, while Trp-212 is within a protein pocket which is hydrophobic (subdomain II 342 A). The Trp-214 in human serum albumin (HSA) is located similarly to Trp-212 in BSA ${ }^{36-38 .}$

343 The quenching effect of Api on fluorescence intensity of serum albumins (BSA and HSA) has 344 been studied previously ${ }^{36,39-42}$ but there is no data related to its behavior in a nanoparticulate 345 system. Studies have shown that the increasing concentration of Api resulted in a decrease in 346 the fluorescence emission intensity of serum albumin solutions. This was mainly attributed to 347 complex formation (static quenching), however, it could be dynamic quenching at higher Api 348 concentrations $^{42}$. Nevertheless, all studies concluded that Api most likely binds to the sub349 domain IIA of Site I side with electrostatic and hydrophobic interactions, through which H350 bonds and non-radiative energy transfer can occur. The binding could affect the conformation 351 of Trp micro-region but the secondary structure of serum albumin is not altered ${ }^{36,} 39,41$. 
352 However, the $\mathrm{pH}$ and ionic concentrations (e.g. $\mathrm{NaCl}$ ) can affect the fluorescence quenching 353 on the binding parameters of apigenin to $\mathrm{BSA}^{43}$.

$354 \quad$ Figure 2 demonstrates the fluorescence emission spectra of BSA solution, BSA-NPs 355 and BSA-Api-NPs. The fluorescence intensity of BSA-NPs decreased slightly compared to 356 BSA solution with no obvious shift of the maximum position at $350 \mathrm{~nm}$. It was probably due 357 to the conformational changes of the protein. The significantly lower emission intensity of 358 BSA-Api-NPs indicates that Api could quench the fluorescence of BSA which is also 359 reflected on the 3D projections (Figure 3). All of these findings indicate that Api binds to the 360 Trp region (Trp-212, subdomain II A) but the spectral maximum was not affected therefore 361 hydrophobicity and polarity of the fluorophore residues are not altered. It was concluded that 362 Api can be bound to the Trp region of serum albumin nanoparticles similarly to the solutions.

\subsection{Characterization of spray-dried BSA-Api-NPs}

\subsubsection{Determination of residual moisture}

Moisture content is mainly influenced by the spray drying conditions. Increased heat energy availability provided by regulating inlet air temperature and aspirator capacity allows more efficient drying, thus resulting in the lower moisture content demonstrated. However, degradation of heat sensitive materials such as proteins may occur; therefore inlet air temperature should be kept below $120^{\circ} \mathrm{C}^{44}$. The water content is also affected by the type of excipients and the ratio with the nanoparticles ${ }^{45}$. Moisture content is an important factor that can significantly influence the aerodynamic properties of aerosols. It can change the surface of particles, promote aggregation and influence the crystallinity of the spray-dried samples ${ }^{44}$. In this study, the residual water content was determined by using Karl Fisher titration. All formulations had relatively low moisture content which followed the rank order of L-leucine $(4.11 \pm 0.21 \%, \mathrm{w} / \mathrm{w})<$ excipient-free $(4.55 \pm 0.49 \%, \mathrm{w} / \mathrm{w})<\operatorname{lactose}(5.8 \pm 0.36 \%, \mathrm{w} / \mathrm{w})$ containing products. These results demonstrate that the optimized outlet air temperature 
377 (around $65^{\circ} \mathrm{C}$ ) was suitable for serum albumin. The L-leucine containing formulation had the

378 lowest water content due to the low hygroscopic behavior of this amino acid ${ }^{46,47}$. The low

379 moisture content can potentially improve the flowability and consequently enhance lung 380 deposition due to reduced aggregation as expected. Storage conditions are also important, e.g.

381 the spray-dried amorphous lactose particles could transform into crystals easily in humidity 382 above $30 \%{ }^{48}$.

\section{$383 \quad 3.2 .2$. Fourier-Transform Infrared Spectroscopy (FT-IR)}

384 FTIR analysis allows a quick and efficient identification of the compounds and by 385 their functional groups and bond vibrations. In the spectrum of raw Api, the following 386 characteristic regions were observed: $2710-2580 \mathrm{~cm}^{-1} \mathrm{O}-\mathrm{H}$ bond, $1730-1680 \mathrm{~cm}^{-1} \mathrm{C}=\mathrm{O}$ stretch 387 and $1450-1380 \mathrm{~cm}^{-1} \mathrm{C}-\mathrm{H}$ bend. A broad peak observed at $3300 \mathrm{~cm}^{-1}$ can be attributed to O-H 388 stretching and those bands at $1600-1400 \mathrm{~cm}^{-1}$ (C-C stretch in ring) and 900-675 $\mathrm{cm}^{-1}(\mathrm{C}-\mathrm{H}$ 389 'oop') can be assigned to the aromatic group (Figure 4 A). In the spectrum of BSA protein, 390 the amide I band at $1635 \mathrm{~cm}^{-1}$ (mainly $\mathrm{C}=\mathrm{O}$ stretch) and amide II band at $1530-1500 \mathrm{~cm}^{-1}(\mathrm{C}-$ $391 \mathrm{~N}$ stretching and $\mathrm{N}-\mathrm{H}$ bend) can be seen. The medium broad peak at $3276 \mathrm{~cm}^{-1}$ corresponds to 392 bonded N-H stretch of amide and a smaller band at $1057 \mathrm{~cm}^{-1}$ is the C-N stretch of aliphatic 393 amine. In the spectra of the excipients-free formulation, the characteristic amide bands of 394 BSA can be seen and peak at $830 \mathrm{~cm}^{-1}$ indicating the presence of Api (aromatic) which is an 395 indirect confirmation of Api encapsulation on BSA-NPs. Conformational changes can be 396 suggested due to the lack of the peak of aliphatic amine.

397 The spectra of raw Api, BSA, lactose and lactose containing product are displayed on Figure $3984 \mathrm{~B}$. In the spectra of lactose there is also a broad band around $3300 \mathrm{~cm}^{-1}$ indicating the 399 stretching vibration of hydroxyl group. A weak band at $1654 \mathrm{~cm}^{-1}$ is the bending vibration of 400 the crystalline water and peaks at $1200-1070 \mathrm{~cm}^{-1}$ demonstrate the stretching vibration of C401 O-C in the glucose and galactose. The spectrum of amorphous lactose has the less number and 
402 defined peaks and therefore it could be distinguished from the crystalline spectrum ${ }^{49}$. The 403 characteristic broad band at $3300 \mathrm{~cm}^{-1}$ in the spectrum of spray-dried product could originate 404 from the residual water content that is further supported by the Karl Fischer titration data 405 (lactose containing product had the highest water content). Similarly to the spectrum of 406 excipients-free formulation, the amide bands of BSA and a small peak of Api could be 407 observed. The peaks at $1200-1070 \mathrm{~cm}^{-1}$ demonstrate the lactose content and the amorphous 408 state could be assumed.

409 Functional groups of L-leucine lead to its characteristic spectrum (Figure 4 C). The strong 410 band in the region of $2970-2910 \mathrm{~cm}^{-1}$ can be accounted for the aliphatic C-H stretching. The 411 bonded N-H stretch is present in the region of $2600-2450 \mathrm{~cm}^{-1}$. The $\mathrm{NH}_{2}$ bending and the C$412 \mathrm{~N}$ skeletal vibration appear in the regions of $1595-1550 \mathrm{~cm}^{-1}$ and $1250-1020 \mathrm{~cm}^{-1} 50$. The 413 presence of BSA characteristic peaks on the spray-dried formulation could be mainly 414 observed with and a small peak of Api but the major characteristic peaks of L-leucine are 415 obscured.

\section{$416 \quad 3.2 .3$. X-ray powder diffraction (XRPD)} XRPD is considered to be the most accurate method to study crystalline structure ${ }^{51}$. The combined XRPD diffractograms of Api and spray-dried formulations are presented in Figure 5. The characteristic narrow diffraction peaks of Api are due to the crystalline state of the commercially available material. In comparison, broad diffuse peaks could be observed in 421 the diffractograms of the spray-dried formulations suggesting the amorphous state of BSA422 Api-NPs. The observed XRD patterns of spray-dried L-leucine and lactose were consistent 423 with literature ${ }^{48,52,53}$.

\section{3.2.4. Differential scanning calorimetry analysis (DSC)}

425 The DSC curves of raw Api, excipents, physical mixtures and spray-dried 426 formulations were studied to examine crystallinity. As seen in Figure 6, there is only one 
427 sharp endothermic peak at $360{ }^{\circ} \mathrm{C}$ indicating the melting point of raw Api; no impurities were 428 observed. Drug free albumin exhibited two broad peaks with onset values of $220{ }^{\circ} \mathrm{C}$ and 310 $429{ }^{\circ} \mathrm{C}$. The evaporation of residual water occurred at $50-120{ }^{\circ} \mathrm{C}$. The melting point of Api on the 430 thermograms of raw material and physical mixtures corresponds to the crystalline habitus. In 431 the thermograms of physical mixtures on Figure $6 \mathbf{B}$ the endothermic peak at $140{ }^{\circ} \mathrm{C}$ 432 indicating the crystalline lactose ${ }^{54}$ and the sublimation of L-leucine crystals occurred at 200$433230{ }^{\circ} \mathrm{C}$ (Figure 6 C) ${ }^{55}$. However, in each spray-dried formulation the absence of endotherms 434 confirms the loss of crystallinity. No peak could be observed around $360{ }^{\circ} \mathrm{C}$ indicating that 435 Api is in amorphous state due to the spray drying process which is in agreement with the 436 XRPD diffractograms. The amorphous form generated may result higher solubility of the 437 powders and dissolution of apigenin in lung fluids.

\subsubsection{Aerosol particle size analysis and redispersibility in water}

Dry powder formulations of BSA-Api-NPs were prepared with the aim of studying the 440 influence of excipients on the particle size and aerodynamic behavior. The deposition of 441 aerosols is significantly affected by particle size which should be small enough to pass 442 through the upper airways and large enough to avoid exhalation ${ }^{56}$. Gravitational 443 sedimentation is the main driving force for deposition of a nanoparticulate system in the lung 444 due to the formation of aggregates in the micrometer size range. Particle geometry and surface 445 properties also play a significant role in reaching the bronchioles ${ }^{22,32}$. It is well known that 446 particles can be deposited efficiently deeper in the lung if their aerodynamic diameter is in the 447 range of 1-5 $\mu \mathrm{m}$ and only those with 1-3 $\mu \mathrm{m}$ can reach the respiratory zone $\mathrm{e}^{57}$. Particles, larger 448 than $5 \mu \mathrm{m}$ tend to deposit in the oropharynx and the mucociliary clearance plays a role in 449 clearing the particles towards the pharynx. However, very small particles, less than $1 \mu \mathrm{m}$ are 450 usually exhaled because of the low inertia ${ }^{58,59}$. Mucociliary clearance is the part of the natural 451 defense mechanism of the lung as well as the phagocytosis of macrophages in the alveolar 
452 region. The aerosol particle size was determined by Sympatec HELOS laser diffractometer

453 (Table 1). The excipient-free and lactose containing products have similar sizes while spray 454 drying with L-leucine produced the smallest particles $\left(\mathrm{D}_{50}=2.473 \mu \mathrm{m}\right)$. In all cases, the 455 particle size could ensure the highest probability of delivery of apigenin into the respiratory 456 zone.

457 Following the re-dispersion of spray powders formulations in distilled water, the size of the 458 particles was preserved in the nanometer size range: without excipient $(358.9 \pm 5.3 \mathrm{~nm}$, PDI: $4590.315 \pm 0.013)$, lactose $(366.1 \pm 4.8 \mathrm{~nm}$, PDI: $0.382 \pm 0.014)$ and L-leucine $(343.7 \pm 2.9$, PDI: $460 \quad 0.316 \pm 0.011)$ containing products. The spray drying has no significant effect on the average 461 size of the particles suggesting the deposition of apigenin containing nanoparticles in the lung 462 fluid. Moreover, the excipients did not affect adversely the particle size.

\subsubsection{Solubility and Drug release studies of BSA-Api formulations}

The solubility of apigenin in nanoparticles was investigated in PBS buffer and mSLF (Figure 7 A). The results showed that the solubility was slightly increased in mSLF media (82-98\% within 5 minutes), however, it was high in PBS buffer as well (79-95\% within 5 minutes). These data indicated that the solubility of apigenin could be highly enhanced by BSA nanoparticles in aqueous medium. Nevertheless, the dispersibility enhancers could play a role in the solubility. In case of excipient free formulation, $91 \%$ of the encapsulated apigenin was dissolved in mSLF within 5 minutes. Formulation prepared with lactose increased the solubility rate up to $98 \%$, however, it was slower ( $82 \%)$ when using L-leucine and completed within 2 hours. These results could be attributed to the solubility of the excipients themselves: lactose has very good water solubility, but L-leucine possess a low solubility in water .

The apigenin release from the spray dried BSA-Api NPs was investigated with Franz cell apparatus. It is a well known device for the dissolution of semisolid dosage forms and 
477 approved by the USP (United States Pharmacopeia). However, there is no standardized 478 method for inhaled powders, Franz cell could be one of the alternative choices due to 479 simulating the diffusion controlled air-liquid interface of the lung. On the contrary, it has 480 some limitations such as small air bubbles under the contact area of membrane to dissolution 481 medium, wide range of standard deviation or the recovery usually around maximum $90 \%{ }^{61}$. 482 Based on the solubility measurements, mSLF was applied. The cumulative dissolution curves 483 of the prepared formulations are shown in Figure 7 B. As expected, the dissolution was 484 affected by the co-spray dried excipients. Lactose containing product resulted the fastest and 485 highest apigenin release due to the excellent water solubility. This enhancement of the 486 dissolution is supported by previously published data ${ }^{62}$. In contrast, the dissolution rate was 487 decreased when L-leucine was applied. The coating layer of L-leucine slowed down the 488 dissolution of apigenin which could be well observed in the dissolution curve. The low water 489 solubility of L-leucine is able to hinder the dissolution of the drug which was published 490 previously $^{60}$. These results suggest that the excipients play an important role in the solubility 491 and the dissolution as well.

$492 \quad 3.2 .7$. Aerosol delivery of BSA-Api formulations

493 Particles can be taken up by alveolar macrophages which influences the therapeutic 494 outcome. Those nanoparticles which are soluble and above $200 \mathrm{~nm}$ are able to escape from 495 the macrophages therefore exhibit sustained therapeutic effect ${ }^{63}$. The lung deposition and 496 therefore the efficacy of the inhaled therapeutics are governed by their aerosol properties ${ }^{56}$. 497 Manufacturing respirable nanoparticles could be produced by aggregation in the favorable 498 size range or their incorporation into microparticles $(1-5 \mu \mathrm{m})^{26}$. Lactose monohydrate is a 499 well-known, traditional carrier for improving the performance of inhaled products; however, 500 it is influenced by physicochemical properties and interaction with the active ingredient ${ }^{64,65}$. 501 It is the only FDA approved carrier and has also been shown to be a potential excipient for 
502 protein encapsulation ${ }^{27,65}$. Recently, novel materials such as specific amino acids have been 503 developed for pulmonary formulations ${ }^{26}$ and L-leucine is one of the most effective 504 dispersibility enhancer among them ${ }^{47}$. Previous studies proved that $5 \%(\mathrm{w} / \mathrm{w})$ L-leucine 505 improved the aerosol performance of raw naringin ${ }^{66}$ and inclusion up to $15 \%(\mathrm{w} / \mathrm{w})$ L-leucine 506 resulted higher ED and FPF of powder formulation of gentamicin ${ }^{46}$.

507 In this study in vitro aerosol properties of three different dry powders formulations 508 were evaluated using the NGI which is regarded as an optimal instrument for analysis of 509 aerodynamic behavior of aerosol formulations for pulmonary drug delivery ${ }^{67}$ according to 510 European and US Pharmacopeias. The obtained data and deposition pattern are presented in 511 Table 2 and in Figure 8. It can be seen that more than $90 \%$ of apigenin could be recovered 512 from the NGI which is in the acceptable pharmacopeia range (75-125\%). The ED ranged 513 between $91-96 \%$ indicating good flowability and high dispersibility of the powders. L-leucine 514 containing formulation had the highest ED as it could improve significantly the flowability of 515 the powders ${ }^{47,53}$. Figure 8 shows the amount of Api deposited on the throat, device and 516 stages 1-7 expressed as a percentage of the total amount of recovered powder. All formulation 517 exhibited increased deposition in Stage 2 - 4 indicating enhanced drug delivery to the alveolar 518 regions. As expected, improved aerosol performance and deposition (Stage 3 and 4) could be 519 observed when L-leucine was used an as excipient. The FPF is one of the key parameters in 520 aerosol delivery and should be as high as possible ${ }^{68}$. In this study, the FPF values ranged 521 between $58-66 \%$, suggesting that the particles could be delivered into the peripheral regions. 522 Spray drying of nanoparticles in the presence of L-leucine resulted higher FPF value (66\%) 523 due to the improved surface properties and morphology of the particles ${ }^{65}$. In general, MMAD 524 values $<5 \mu \mathrm{m}$ are for pulmonary lung delivery and between 2-3 $\mu \mathrm{m}$ are optimal for deep lung 525 deposition ${ }^{56}$. In each cases, the calculated mass median aerodynamic diameter (MMAD) data 526 were in agreement with the physical diameter size of the particles measured by laser 
527 diffractometer. The data obtained $(<5 \mu \mathrm{m})$ support good dispersibility of the particles into the

528 lower airways and the deep lung. Therefore local delivery to the alveoli could be assured by

529 both excipient-free and lactose formulations generated (MMAD $3.2 \mu \mathrm{m}$ and $3.1 \mu \mathrm{m}$ ).

530 Moreover, formulation with L-leucine (MMAD $2.1 \mu \mathrm{m})$ would be more optimal for deep lung

531 deposition. The size distribution of an aerosol is described best by GSD ${ }^{69}$. Based on the GSD

532 data obtained, the L-leucine containing formulation had the narrowest size distribution (1.8

$533 \mu \mathrm{m})$ but that of the others was also in the acceptable range $(<3 \mu \mathrm{m})$.

534 The overall values demonstrate that the particles of each dry powder nanoparticle formulation

535 are in the favorable aerodynamic size range, possess good dispersibility properties and 536 particle deposition. Therefore BSA-NPs is an attractive delivery system for pulmonary drug

537 delivery. We demonstrated that L-leucine improved better the aerosolization properties of

538 BSA-Api-NPs than lactose monohydrate. Therefore it can be concluded that the use of 539 excipients influence the aerosol performance of nanoparticles.

$540 \quad$ 3.2.8. Particle morphology

541 SEM analysis was conducted to investigate the morphology of the powders (Figure 9

542 A and B). It is well known that the morphology of the particles is strongly affected by the

543 solubility of the components and the nature of the excipients ${ }^{46,47}$. The commercially available

544 Api was a crystalline powder featuring needle-shaped crystals. The excipient-free spray-dried

545 nanoparticles exhibited spherical shape and smooth or wrinkled surface. Particles of lactose

546 containing product had raisin-like surface and some of the particles were larger in accordance

547 with the laser diffraction particle size analysis. Powders prepared with L-leucine comprised

548 small and collapsed particles with strongly corrugated surface. The low aqueous solubility of

549 L-leucine leading to a shell on the surface of the droplet which interfere with the diffusion of

550 water therefore corrugated particles could form. This outcome was consistent with previous

551 observations ${ }^{46,53,70}$. Corrugated surface improves the dispersibility of the dry powder 
552 formulations and enhance respirability due to the reduced interparticulate cohesion (Van der

553 Waals forces) which is beneficial for particles intended for inhalation ${ }^{71}$.

$554 \quad$ 3.3. Antioxidant activity

555 Owing to its reproducibility and comparability, the $\mathrm{DPPH}^{*}$ assay is an established 556 method for investigating the antioxidant properties of natural compounds. Due to the $\mathrm{H}$ 557 donating ability of the antioxidants, a stable reduced DPPH-H molecule can form. The 558 reaction can be seen visually and the detection can be carried out using UV-Vis 559 spectrophotometer ${ }^{72,73}$. Previous studies confirmed that Api is able to scavenge the DPPH 560 free radial even in nanoscale delivery formulation ${ }^{74,75}$. In general, the scavenging activity is 561 influenced by concentration and structural features like hydrogen donating ability, position 562 and the degree of hydroxylation ${ }^{76,77}$. In order to calculate the exact concentration of 563 remaining $\mathrm{DPPH}^{*}$ in the samples a calibration curve was plotted with $\mathrm{R}^{2}=0.9999$. The time 564 required to reach the steady state was estimated to be 120 minutes, and the slow reaction 565 kinetic of Api has been reported ${ }^{74}$. The discoloration of the deep purple DPPH ${ }^{\bullet}$ free radical 566 indicate the antioxidant properties of free and encapsulated Api. The inhibition of free 567 radicals by the prepared spray-dried formulations were compared to the empty BSA-NPs, 568 methanolic Api solution and "empty" nanoparticles (Figure 10). It can be seen that the free 569 and encapsulated Api have similar scavenging activity, moreover, the spray drying did not 570 result in the loss of scavenging activity. It has been reported that serum albumin is a 571 physiological circulating antioxidant in the $\operatorname{body}^{78}$ which is confirmed by the inhibition 572 capacity of the empty BSA-NPs observed. Similar results were reported when encapsulating 573 rutin and keampferol ${ }^{79}$ or quercetin ${ }^{17}$ where the antioxidant activity of the flavonoids are 574 retained by BSA. It can be concluded that the antioxidant activity of Api is preserved, 575 moreover, slightly enhanced by the BSA. 
577 In this study novel apigenin containing albumin nanoparticles were prepared for 578 inhalation against lung injury caused by oxidative stress. Apigenin was recently classified as a

579 BCS II. drug with prominent antioxidant and anti-inflammatory properties in the lung. The 580 obtained results confirmed that incorporation of apigenin into the biocompatible albumin 581 nanoparticles resulted high encapsulation efficiency therefore it could be an attractive tool for 582 the delivery. Moreover, the spray dried nanoparticles possess good ability to re-disperse in 583 aqueous media and size of the particles was preserved in the nanometer size range. The 584 influence of dispersibility enhancers on the physicochemical properties and in vitro 585 pulmonary deposition were investigated and compared to the excipient-free formulation. The 586 obtained in vitro pulmonary depositions proved that the developed BSA-NP dry powders are 587 potentially able to carry apigenin deep in the lung, reaching the respiratory zone. The use of 588 novel excipient amino acid L-leucine resulted enhanced aerodynamic properties over the 589 traditional lactose monohydrate, indicating that the nature of the excipients and morphology 590 of the particles play a significant role in the formulation of nanoparticles for pulmonary 591 delivery. In addition, the solubility and dissolution characteristics of apigenin from 592 nanoparticles were determined in mSLF dissolution media, the co-spray dried excipients 593 played an important role. The dissolution rate was increased by the water soluble lactose and 594 decreased by L-leucine, which has low water solubility. Therefore the use of excipients should 595 be taken into consideration, may not required in case of albumin nanoparticles. We further 596 confirmed that the antioxidant activity is retained, thus the potential of albumin nanoparticles 597 as an effective pulmonary delivery system for flavonoids such as apigenin.

\section{ACKNOWLEDGEMENTS}

599 The authors gratefully acknowledge to Róbert Kovács for providing the SEM pictures at 600 Budapest University of Technology and Economics.

\section{Author Disclosure Statement}


602 The authors declare that there are no conflicts of interest.

603 


\section{REFERENCES}

605 1. Chow C-W, Herrera Abreu MT, Suzuki T, and Downey GP. Oxidative Stress and 606 Acute Lung Injury. Am J Respir Cell Mol Biol. 2003;29:427-431.

607 2. Chen XW, Serag ES, Sneed KB, and Zhou SF. Herbal bioactivation, molecular targets 608 and the toxicity relevance. Chem Biol Interact. 2011;192:161-176.

609 3. Patel D, Shukla S, and Gupta S. Apigenin and cancer chemoprevention: progress, 610 potential and promise (review). Int J Oncol. 2007;30:233-245.

611 4. Kim SJ, Jeong HJ, Moon PD, Lee KM, Lee HB, Jung HJ, Jung SK, Rhee HK, Yang 612 DC, Hong SH, and Kim HM. Anti-inflammatory activity of gumiganghwaltang 613 through the inhibition of nuclear factor-kappa B activation in peritoneal macrophages. 614 Biol Pharm Bull. 2005;28:233-237.

615 5. Kowalski J, Samojedny A, Paul M, Pietsz G, and Wilczok T. Effect of apigenin, 616 kaempferol and resveratrol on the expression of interleukin-1beta and tumor necrosis 617 factor-alpha genes in J774.2 macrophages. Pharmacol Rep. 2005;57:390-394.

618 6. Lee J-H, Zhou H, Cho S, Kim Y, Lee Y, and Jeong C. Anti-inflammatory mechanisms 619 of apigenin: inhibition of cyclooxygenase-2 expression, adhesion of monocytes to 620 human umbilical vein endothelial cells, and expression of cellular adhesion molecules. 621 Archives of Pharmacal Research. 2007;30:1318-1327.

622 7. Chen L and Zhao WEI. Apigenin protects against bleomycin-induced lung fibrosis in 623 rats. Exp Ther Med. 2016;11:230-234.

624 8. Luan RL, Meng XX, and Jiang W. Protective Effects of Apigenin Against Paraquat625 Induced Acute Lung Injury in Mice. Inflammation. 2016; 39:752-758.

626 9. Basios N, Lampropoulos P, Papalois A, Lambropoulou M, Pitiakoudis MK, Kotini A, 627 Simopoulos $\mathrm{C}$, and Tsaroucha AK. Apigenin Attenuates Inflammation in 
628

629

630

631

632

633

634

635

636

637

638

639

640

641

642

643

644

645

646

647

648

649

650

651

Experimentally Induced Acute Pancreatitis-Associated Lung Injury. J Invest Surg. 2015;2:1-7.

10. Patil R, Babu RL, Naveen Kumar M, Kiran Kumar KM, Hegde S, Ramesh G, and Chidananda Sharma S. Apigenin inhibits PMA-induced expression of proinflammatory cytokines and AP-1 factors in A549 cells. Mol Cell Biochem. 2015;403:95-106.

11. Wang J, Liu Y-T, Xiao L, Zhu L, Wang Q, and Yan T. Anti-Inflammatory Effects of Apigenin in Lipopolysaccharide-Induced Inflammatory in Acute Lung Injury by Suppressing COX-2 and NF-kB Pathway. Inflammation. 2014;37:2085-2090.

12. Zhang J, Liu D, Huang Y, Gao Y, and Qian S. Biopharmaceutics classification and intestinal absorption study of apigenin. Int J Pharm. 2012;436:311-317.

13. Ajazuddin and Saraf S. Applications of novel drug delivery system for herbal formulations. Fitoterapia. 2010;81:680-689.

14. Elzoghby AO, Samy WM, and Elgindy NA. Albumin-based nanoparticles as potential controlled release drug delivery systems. J Control Release. 2012;157:168-182.

15. Hu Y-J, Liu Y, Sun T-Q, Bai A-M, Lü J-Q, and Pi Z-B. Binding of anti-inflammatory drug cromolyn sodium to bovine serum albumin. Int J Biol Macromolec. 2006;39:280285.

16. Fasano M, Curry S, Terreno E, Galliano M, Fanali G, Narciso P, Notari S, and Ascenzi P. The extraordinary ligand binding properties of human serum albumin. IUBMB Life. 2005;57:787-796.

17. Fang $\mathrm{R}$, Hao $\mathrm{R}$, Wu $\mathrm{X}$, Li $\mathrm{Q}$, Leng $\mathrm{X}$, and Jing $\mathrm{H}$. Bovine Serum Albumin Nanoparticle Promotes the Stability of Quercetin in Simulated Intestinal Fluid. J Agr Food Chem. 2011;59:6292-6298. 
652 18. He X, Xiang N, Zhang J, Zhou J, Fu Y, Gong T, and Zhang Z. Encapsulation of 653 teniposide into albumin nanoparticles with greatly lowered toxicity and enhanced 654 antitumor activity. Int J Pharm. 2015;487:250-259.

655 19. Muralidharan P, Malapit M, Mallory E, Hayes Jr D, and Mansour HM. Inhalable 656 nanoparticulate powders for respiratory delivery. Nanomedicine: NBM. 2015;11:1189$657 \quad 1199$

658 20. Malcolmson RJ and Embleton JK. Dry powder formulations for pulmonary delivery. $659 \quad$ Pharm Sci Technol To. 1998;1:394-398.

660 21. Stegemann S, Kopp S, Borchard G, Shah VP, Senel S, Dubey R, Urbanetz N, Cittero 661

662

663

664 M, Schoubben A, Hippchen C, Cade D, Fuglsang A, Morais J, Borgström L, Farshi F, Seyfang KH, Hermann R, van de Putte A, Klebovich I, and Hincal A. Developing and advancing dry powder inhalation towards enhanced therapeutics. Eur J Pharm Sci. 2013;48:181-194.

22. Yang W, Peters JI, and Williams Iii RO. Inhaled nanoparticles-A current review. Int J Pharm. 2008;356:239-247.

667

668

669

670

671

672

673

674

25. Swarbrick J. Encyclopedia of Pharmaceutical Technology. Informa Healthcare:London; 2007; 671 - 1434.

675

26. Mansour $\mathrm{HM}$, Rhee $\mathrm{YS}$, and $\mathrm{Wu} \mathrm{X}$. Nanomedicine in pulmonary delivery. Int $J$ 676 Nanomedicine. 2009;4:299-319. 
677 27. Shoyele SA and Cawthorne S. Particle engineering techniques for inhaled

678

679

680

681

682

683

684

685

686

687

688

689

690

691

692

693

694

695

696

697

698

699

700

701 biopharmaceuticals. Adv Drug Deliv Rev. 2006;58:1009-1029.

28. Sung JC, Pulliam BL, and Edwards DA. Nanoparticles for drug delivery to the lungs. Trends Biotechnol. 2007;25:563-570.

29. Desai NP, Tao C, Yang A, Louie L, Yao Z, Soon-Shiong P, and Magdassi S. Protein stabilized pharmacologically active agents, methods for the preparation thereof and methods for the use thereof. Google Patents, 2004. US6749868 B1

30. Son YJ, McConville JT. Development of a standardized dissolution test method for inhaled pharmaceutical formulations. Int J Pharm. 2009;382:15-22.

31. Hatano T. KH, Yasuhara T., Okuda T. 2 new flavonoids and other constituents in licorice root-their relative astringency and radical scavenging effects. Chem Pharm Bull. 1988;36:2090-2097.

32. Paranjpe $\mathrm{M}$ and Muller-Goymann CC. Nanoparticle-mediated pulmonary drug delivery: a review. Int J Mol Sci. 2014;15:5852-5873.

33. Kim TH, Jiang HH, Youn YS, Park CW, Tak KK, Lee S, Kim H, Jon S, Chen X, and Lee KC. Preparation and characterization of water-soluble albumin-bound curcumin nanoparticles with improved antitumor activity. Int J Pharm. 2011;403:285-291.

34. Wei Y, Li L, Xi Y, Qian S, Gao Y, and Zhang J. Sustained release and enhanced bioavailability of injectable scutellarin-loaded bovine serum albumin nanoparticles. Int J Pharm. 2014;476:142-148.

35. Valeur B. Molecular fluorescence principles and applications. Wiley-VCH: Weinheim; 2002; 73-124.

36. Yuan J-L, lv Z, Liu Z-G, Hu Z, and Zou G-L. Study on interaction between apigenin and human serum albumin by spectroscopy and molecular modeling. $J$ Photochem Photobiol A. 2007;191:104-113. 
702 37. Naveenraj S and Anandan S. Binding of serum albumins with bioactive substances $703 \quad$ Nanoparticles to drugs. J Photochem Photobiol C. 2013;14:53-71.

704 38. Tayeh N, Rungassamy $\mathrm{T}$, and Albani JR. Fluorescence spectral resolution of 705 tryptophan residues in bovine and human serum albumins. J Pharm Biomed Anal. $706 \quad 2009 ; 50: 107-116$.

707

708

709

710

711

712

713

714

715

716

717

718

719

720

721

722

723

724

725

39. Zhao X-N, Liu Y, Niu L-Y, and Zhao C-P. Spectroscopic studies on the interaction of bovine serum albumin with surfactants and apigenin. Spectrochim Acta A Mol Biomol Spectrosc. 2012;94:357-364.

40. Lin $\mathrm{C}-\mathrm{Z}, \mathrm{Hu} \mathrm{M}, \mathrm{Wu} \mathrm{A}-\mathrm{Z}$, and Zhu C-C. Investigation on the differences of four flavonoids with similar structure binding to human serum albumin. $J$ Pharm Anal. 2014;4:392-398.

41. Cao H, Liu Q, Shi J, Xiao J, and Xu M. Comparing the Affinities of Flavonoid Isomers with Protein by Fluorescence Spectroscopy. Anal Lett. 2008;41:521-532.

42. Shang $\mathrm{Y}$ and $\mathrm{Li} \mathrm{H}$. Studies of the interaction between apigenin and bovine serum albumin by spectroscopic methods. Russ J Gen Chem. 2010;80:1710-1717.

43. Tang L, Jia W, and Zhang D. The effects of experimental conditions of fluorescence quenching on the binding parameters of apigenin to bovine serum albumin by response surface methods. Luminescence. 2014;29:344-351.

44. Stahl K, Claesson M, Lilliehorn P, Linden H, and Backstrom K. The effect of process variables on the degradation and physical properties of spray dried insulin intended for inhalation. Int J Pharm. 2002;233:227-237.

45. Jensen DM, Cun D, Maltesen MJ, Frokjaer S, Nielsen HM, and Foged C. Spray drying of siRNA-containing PLGA nanoparticles intended for inhalation. J Control Release. 2010;142:138-145. 
726 46. Aquino RP, Prota L, Auriemma G, Santoro A, Mencherini T, Colombo G, and Russo

727 P. Dry powder inhalers of gentamicin and leucine: formulation parameters, aerosol 728 performance and in vitro toxicity on CuFi1 cells. Int J Pharm. 2012;426:100-107.

729 47. Yang X-F, Xu Y, Qu D-S, and Li H-Y. The influence of amino acids on aztreonam 730 spray-dried powders for inhalation. Asian J Pharm Sci. 2015;10:541-548.

731

732

733

734

735

736

737

738

739

740

741

742

743

744

745

746

747

748

749

750

48. Wu L, Miao X, Shan Z, Huang Y, Li L, Pan X, Yao Q, Li G, and Wu C. Studies on the spray dried lactose as carrier for dry powder inhalation. Asian J Pharm Sci. 2014;9:336-341.

49. Listiohadi Y, Hourigan J, Sleigh R, and Steele R. Thermal analysis of amorphous lactose and $\alpha$-lactose monohydrate. Dairy Sci Technol. 2009;89:43-67.

50. Němec I and Mička Z. FTIR and FT Raman study of L-leucine addition compound with nitric acid. J Mol Struct. 1999;482-483:23-28.

51. Newman AW and Byrn SR. Solid-state analysis of the active pharmaceutical ingredient in drug products. Drug Discov Today. 2003;8:898-905.

52. Sou T, Kaminskas LM, Nguyen T-H, Carlberg R, McIntosh MP, and Morton DAV. The effect of amino acid excipients on morphology and solid-state properties of multicomponent spray-dried formulations for pulmonary delivery of biomacromolecules. Eur J Pharm Biopharm. 2013;83:234-243.

53. Mangal S, Meiser F, Tan G, Gengenbach T, Denman J, Rowles MR, Larson I, and Morton DAV. Relationship between surface concentration of 1-leucine and bulk powder properties in spray dried formulations. Eur J Pharm Biopharm. 2015;94:160169.

54. Gombás Á, Szabó-Révész P, Kata M, Regdon G, Jr., and Erős I. Quantitative Determination of Crystallinity of $\alpha$-Lactose Monohydrate by DSC. $J$ Therm Anal Calorim. 2002;68:503-510. 
751 55. Raula J, Seppälä J, Malm J, Karppinen M, and Kauppinen EI. Structure and 752 Dissolution of 1-Leucine-Coated Salbutamol Sulphate Aerosol Particles. AAPS 753 PharmSciTech. 2012;13:707-712.

754 56. Yang MY, Chan JGY, and Chan H-K. Pulmonary drug delivery by powder aerosols. $J$ $755 \quad$ Control Release. 2014;193:228-240.

756

757

758

759

760

761

762

763

764

765

766

767

768

769

770

771

772

773

774

775

776

57. Chow AL, Tong HY, Chattopadhyay P, and Shekunov B. Particle Engineering for Pulmonary Drug Delivery. Pharm Res. 2007;24:411-437.

58. Liang Z, Ni R, Zhou J, and Mao S. Recent advances in controlled pulmonary drug delivery. Drug Discov To. 2015;20:380-389.

59. Heyder J, Gebhart J, Rudolf G, Schiller CF, and Stahlhofen W. Deposition of particles in the human respiratory tract in the size range 0.005-15 $\mu \mathrm{m}$. J Aerosol Sci. $1986 ; 17: 811-825$.

60. Raula J, Seppälä J, Malm J, Karppinen M, Kauppinen EI. Structure and dissolution of L-leucine-coated salbutamol sulphate aerosol particles. AAPS PharmSciTech. 2012; 13:707-12.

61. May S, Jensen B, Wolkenhauer M, Schneider M, Lehr CM. Dissolution techniques for in vitro testing of dry powders for inhalation. Pharm Res. 2012;29:2157-66.

62. Knieke C, Azad MA, To D, Bilgili E, Davé RN. Sub-100 micron fast dissolving nanocomposite drug powders. Powder Technol. 2015;271:49-60.

63. Patel B, Gupta N, and Ahsan F. Particle engineering to enhance or lessen particle uptake by alveolar macrophages and to influence the therapeutic outcome. Eur $J$ Pharm Biopharm. 2015;89:163-174.

64. Kou X, Chan LW, Steckel H, and Heng PWS. Physico-chemical aspects of lactose for inhalation. Adv Drug Deliv Rev. 2012;64:220-232.

65. Pilcer G, Wauthoz N, and Amighi K. Lactose characteristics and the generation of the aerosol. Adv Drug Deliv Rev. 2012;64:233-256. 
777 66. Prota L, Santoro A, Bifulco M, Aquino RP, Mencherini T, and Russo P. Leucine 778 enhances aerosol performance of Naringin dry powder and its activity on cystic 779 fibrosis airway epithelial cells. Int J Pharm. 2011;412:8-19.

780 67. Taki M, Marriott C, Zeng X-M, and Martin GP. Aerodynamic deposition of 781 combination dry powder inhaler formulations in vitro: A comparison of three 782 impactors. Int J Pharm. 2010;388:40-51.

783 68. Davis SS. Delivery of peptide and non-peptide drugs through the respiratory tract. 784

71. Chew NY and Chan HK. The role of particle properties in pharmaceutical powder inhalation formulations. $J$ Aerosol Med. 2002;15:325-330.

72. Brand-Williams W, Cuvelier ME, and Berset C. Use of a free radical method to evaluate antioxidant activity. LWT - Food Science and Technology. 1995;28:25-30.

73. Villaño D, Fernández-Pachón MS, Moyá ML, Troncoso AM, and García-Parrilla MC. Radical scavenging ability of polyphenolic compounds towards $\mathrm{DPPH}^{\bullet}$ free radical. Talanta. 2007;71:230-235.

74. Al Shaal L, Shegokar R, and Muller RH. Production and characterization of antioxidant apigenin nanocrystals as a novel UV skin protective formulation. Int $J$ Pharm. 2011;420:133-140. 
801 75. Papay Z.E AI. Study on the antioxidant activity during the formulation of biological 802 active ingredient. European Scientific Journal. 2014;Special Edition 3:252-257.

803 76. Yang J, Guo J, and Yuan J. In vitro antioxidant properties of rutin. LWT - Food $804 \quad$ Science and Technology. 2008;41:1060-1066.

805 77. Natella F, Nardini M, Di Felice M, and Scaccini C. Benzoic and Cinnamic Acid 806 Derivatives as Antioxidants: Structure-Activity Relation. J Agr Food Chem. $807 \quad 1999 ; 47: 1453-1459$.

808 78. Roche M, Rondeau P, Singh NR, Tarnus E, and Bourdon E. The antioxidant properties $809 \quad$ of serum albumin. FEBS Lett. 2008;582:1783-1787.

810 79. Fang R, Jing H, Chai Z, Zhao G, Stoll S, Ren F, Liu F, and Leng X. Study of the 811 physicochemical properties of the BSA: flavonoid nanoparticle. Eur Food Res $812 \quad$ Technol. 2011;233:275-283.

813

814 
815 TABLES

816 Table 1

817 Aerosol particle sizes of spray-dried nanoparticles with Sympatec HELOS laser 818 diffractometer in $\mu \mathrm{m}$.

\begin{tabular}{llll}
\hline & Excipient-free & Lactose & L-leucine \\
\cline { 2 - 4 } ED (\%) & $91.862 \pm 2.735$ & $93.950 \pm 1.046$ & $95.183 \pm 0.667$ \\
FPF (\%) & $65.617 \pm 3.422$ & $58.463 \pm 6.031$ & $66.090 \pm 2.777$ \\
MMAD ( $\boldsymbol{\mu m})$ & $3.210 \pm 0.069$ & $3.130 \pm 0.001$ & $2.123 \pm 0.098$ \\
GSD ( $\boldsymbol{\mu m})$ & $2.823 \pm 0.113$ & $2.270 \pm 0.212$ & $1.887 \pm 0.063$ \\
RD (\%) & $99.1 \pm 5.012$ & $94.7 \pm 4.091$ & $96.3 \pm 2.161$ \\
\hline
\end{tabular}

Table 2

822 Aerodynamic characteristic of spray-dried nanoparticles.

\begin{tabular}{llll}
\hline & Excipient-free & Lactose & L-leucine \\
\cline { 2 - 4 } $\mathbf{D}_{\mathbf{1 0}}$ & $1.033 \pm 0.032$ & $1.020 \pm 0.070$ & $0.843 \pm 0.680$ \\
$\mathbf{D}_{\mathbf{5 0}}$ & $3.030 \pm 0.092$ & $3.107 \pm 0.102$ & $2.473 \pm 0.300$ \\
$\mathbf{D}_{\mathbf{9 0}}$ & $7.110 \pm 0.306$ & $7.117 \pm 0.337$ & $5.287 \pm 0.670$ \\
\hline
\end{tabular}




\section{FIGURE CAPTIONS}

826 Figure 1. Molecular structure of apigenin.

827 Figure 2. Fluorescence emission spectra of BSA solution, BSA nanoparticles and BSA-Api 828 nanoparticles. The excitation wavelength was set to $285 \mathrm{~nm}$ (EM: emission, EXC: $829 \quad$ excitation)

830 Figure 3. Three dimensional fluorescence emission maps and two dimensional contour maps of empty BSA nanoparticles and BSA-Api nanoparticles. Color scale displays the range of observed fluorescence intensities.

Figure 4. A) FT-IR spectra of apigenin (1), BSA (2) and the excipient-free spray-dried BSA-Api nanoparticles (3).

Figure 5. XRPD diffraction pattern of raw apigenin and the formulations.

Figure 6. A) DSC thermograms of apigenin (1), BSA (2) physical mixture (3) and the excipient-free spray dried BSA-Api nanoparticles (4).

B) DSC spectra of apigenin (1), BSA (2), physical mixture (3) and the spray-dried BSA-Api nanoparticles with lactose (4).

C) DSC spectra of apigenin (1), BSA (2), physical mixture (3) and the spray-dried BSA-Api nanoparticles with L-leucine (4).

Figure 7. A) Solubility of spray dried BSA-Api formulations in PBS buffer and modified simulated lung fluid (mSLF).

B) Dissolution of apigenin from the formulations as a function of time in modified simulated lung fluid (mSLF). 
850 Figure 8. NGI deposition pattern of the spray dried BSA-Api formulations.

851 Figure 9. SEM images of raw apigenin (1), excipient-free spray dried BSA-Api 852 nanoparticles (2), spray-dried BSA-Api nanoparticles with lactose (3), spray-dried 853 BSA-Api nanoparticles with L-leucine (4) 20000 x magnification.

854 Figure 10. Radical scavenging activity of Apigenin solution, empty BSA nanoparticles, 855 BSA-Apigenin nanoparticles (NP) and spray-dried nanoparticles (SD) with 856 excipients. The antioxidant activity is expressed as the inhibition of DPPH free 857 radical in percent.

858 To whom correspondence should be addressed:

859 antal.istvan@pharma.semmelweis-univ.hu

860 Department of Pharmaceutics

861 Semmelweis University

862 Hőgyes E. Street 7-9,

863 H-1092 Budapest, Hungary

864 Tel/Fax: +3612017-0914; Tel.: +361476-3600/53066, 53087 


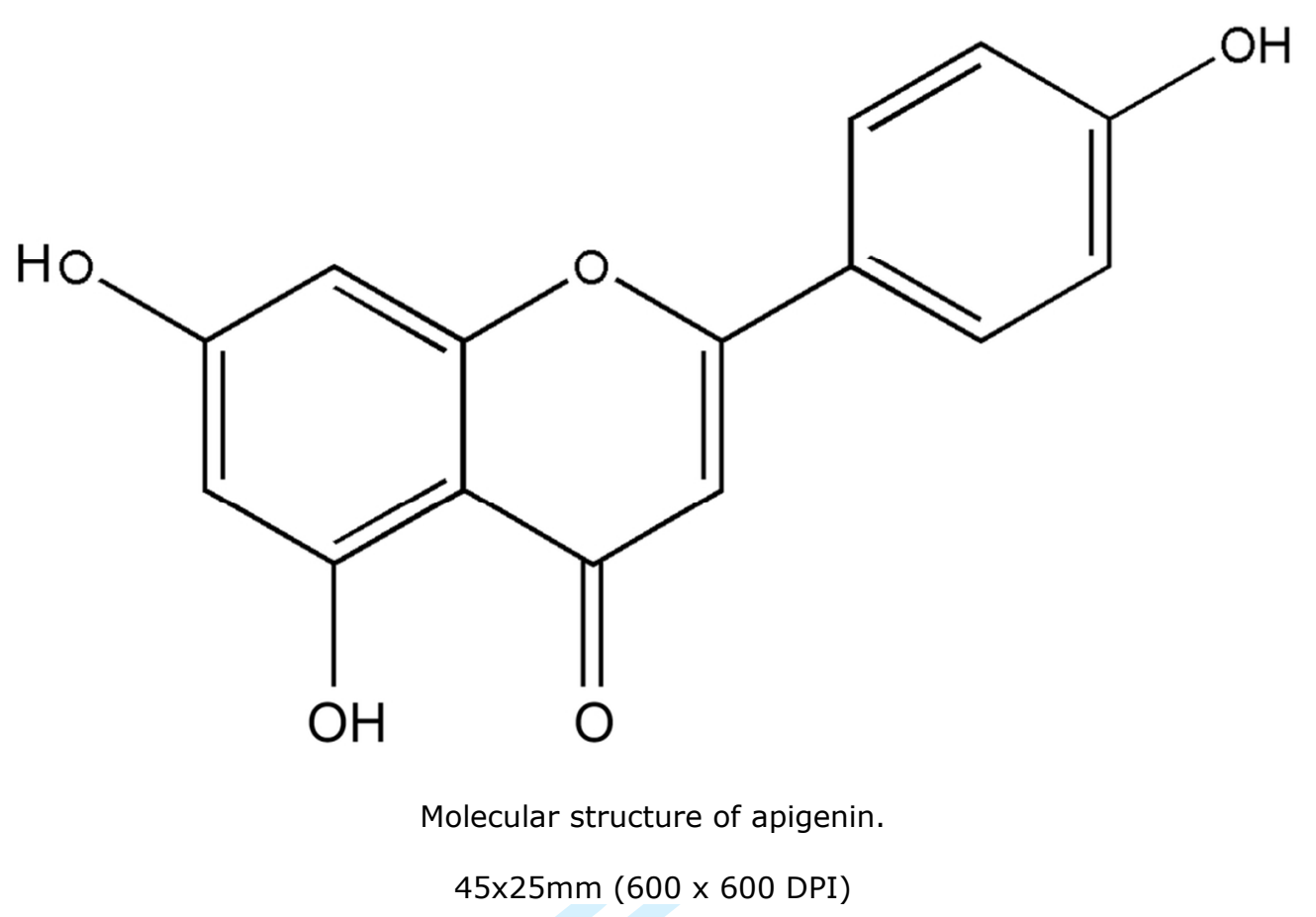

Mary Ann Liebert Inc., 140 Huguenot Street, New Rochelle, NY 10801 


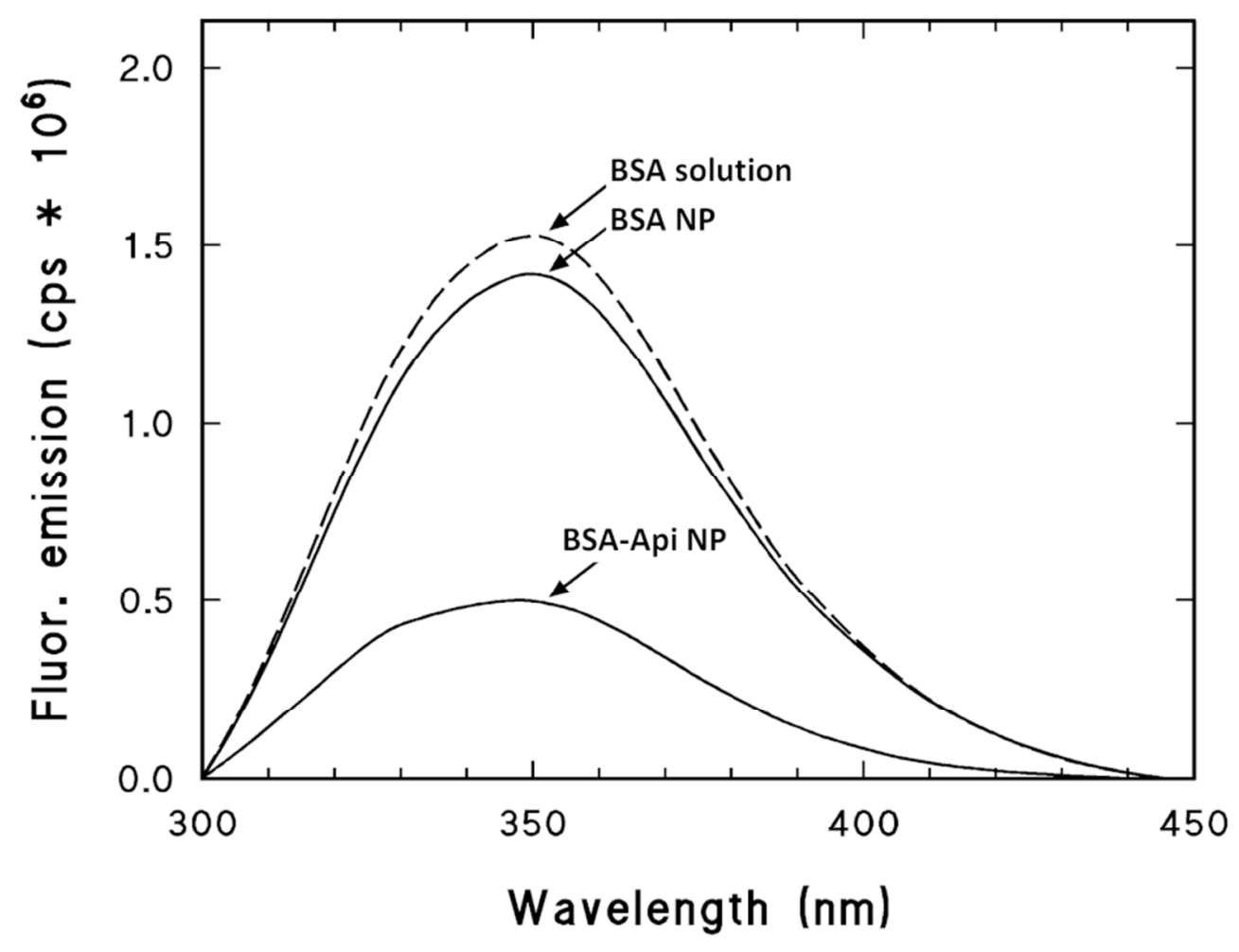

Fluorescence emission spectra of BSA solution, BSA nanoparticles and BSA-Api nanoparticles. The excitation wavelength was set to $285 \mathrm{~nm}$ (EM: emission, EXC: excitation)

$80 \times 60 \mathrm{~mm}(300 \times 300 \mathrm{DPI})$ 
Three dimensional fluorescence emission maps and two dimensional contour maps of empty BSA nanoparticles and BSA-Api nanoparticles. Color scale displays the range of observed fluorescence intensities. 


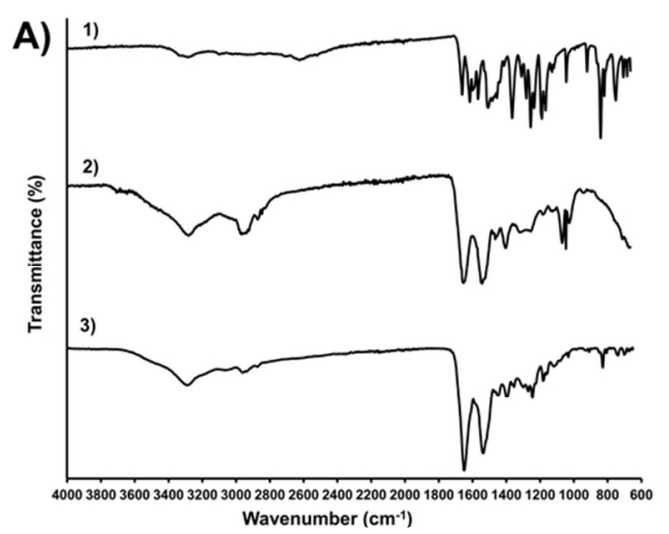

B)

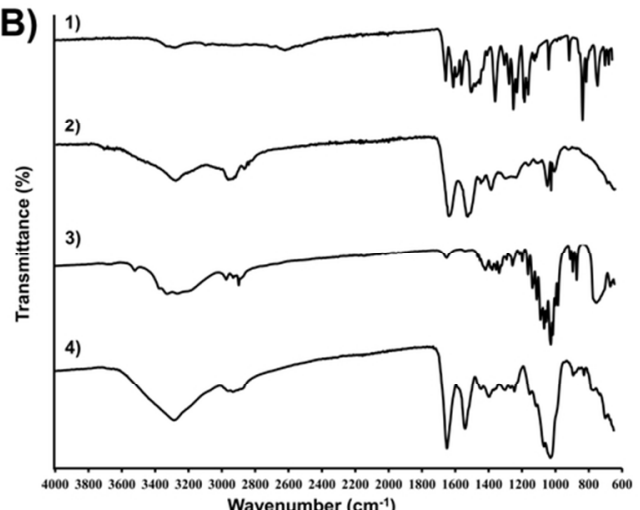

Wavenumber $\left(\mathrm{cm}^{-1)}\right.$

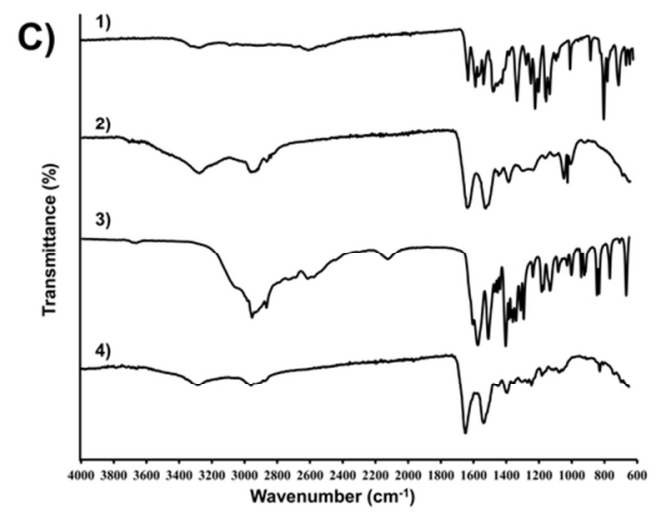

A) FT-IR spectra of apigenin (1), BSA (2) and the excipient-free spray-dried BSA-Api nanoparticles (3).

B) FT-IR spectra of apigenin (1), BSA (2), Lactohale ${ }^{\circledR}(3)$ and the spray-dried BSA-Api nanoparticles with Lactohale $\circledast$ (4).

C) FT-IR spectra of apigenin (1), BSA (2), L-leucine (3) and the spray-dried BSA-Api nanoparticles with Lleucine (4).

$42 \times 34 \mathrm{~mm}(600 \times 600 \mathrm{DPI})$ 


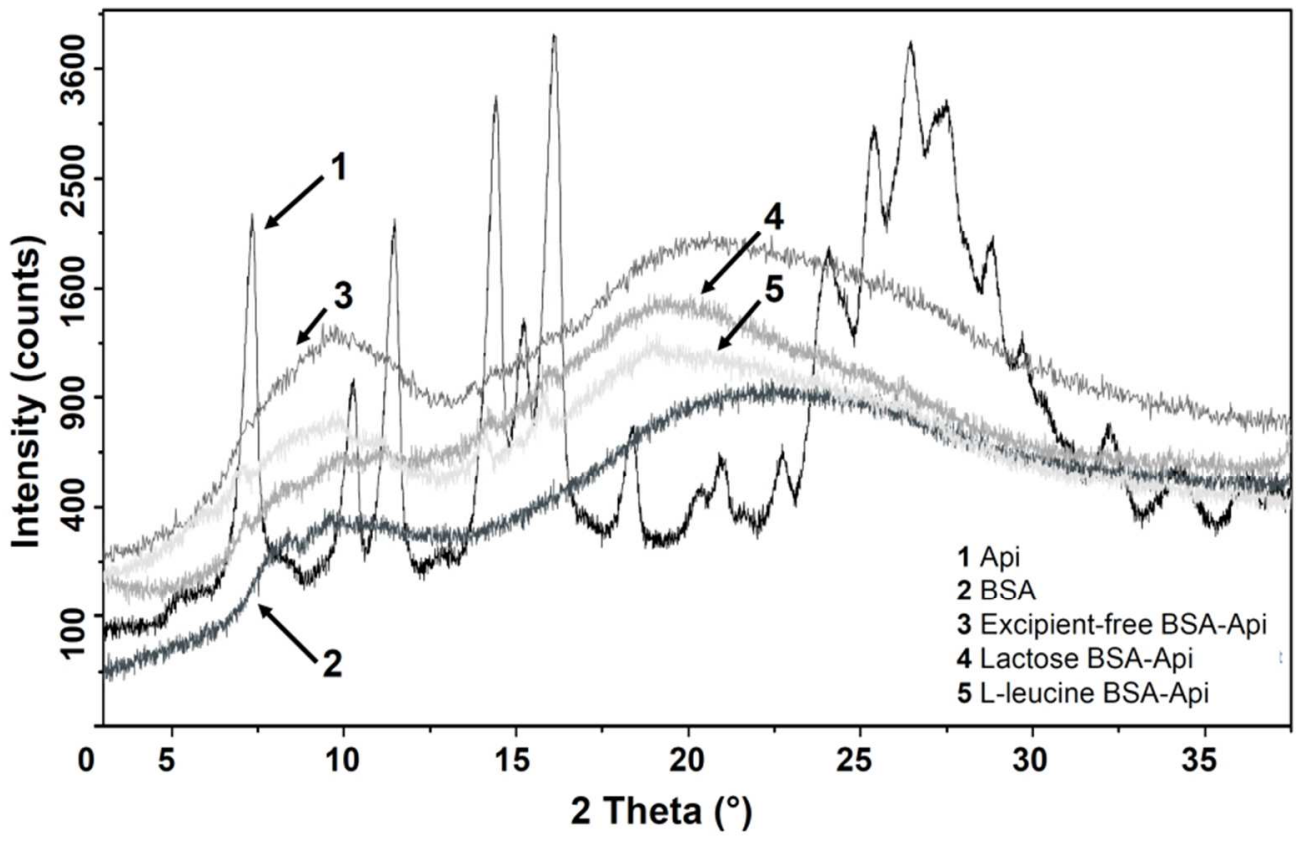

XRPD diffraction pattern of raw apigenin and the formulations.

$80 \times 52 \mathrm{~mm}(300 \times 300$ DPI $)$ 

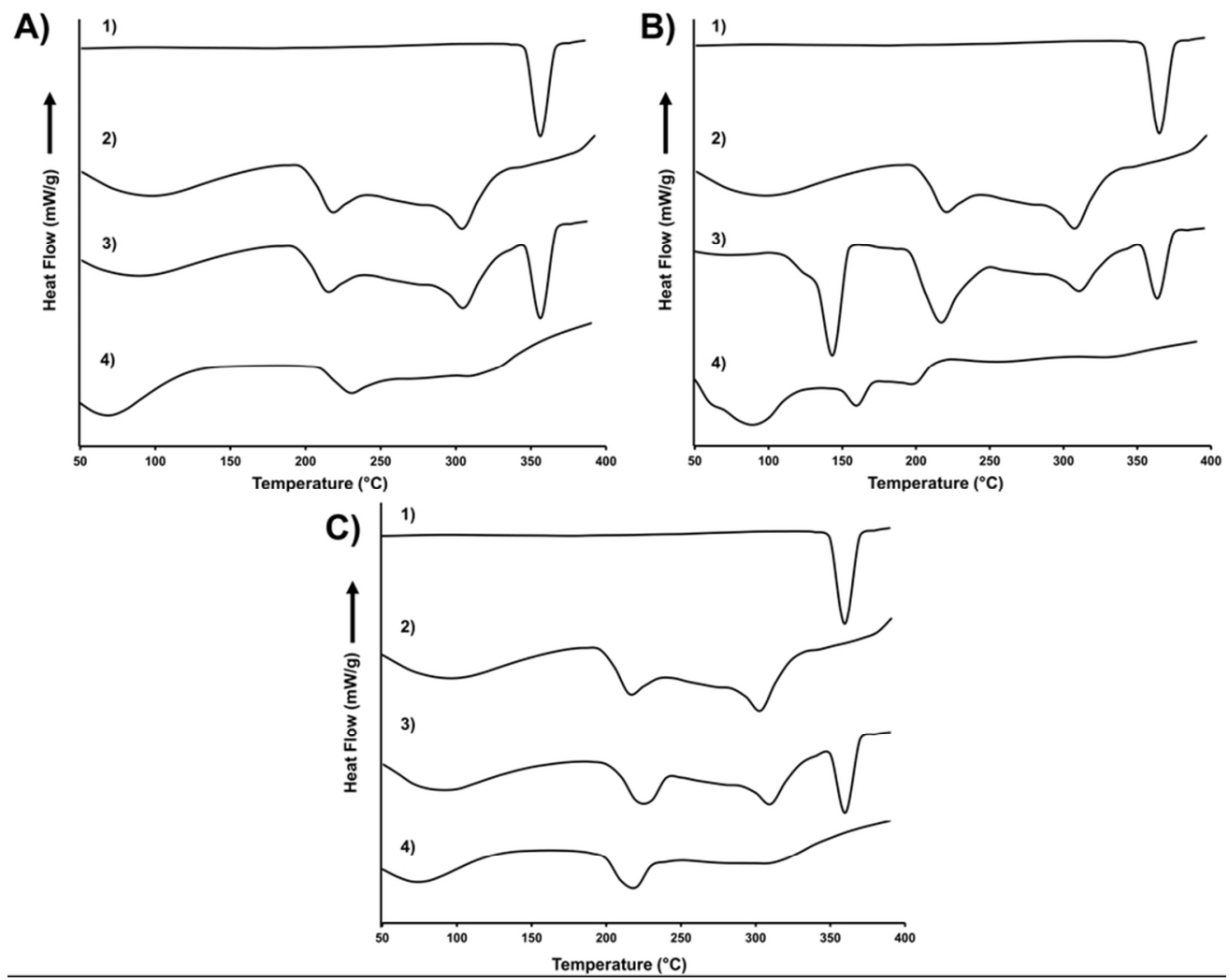

A) DSC thermograms of apigenin (1), BSA (2) physical mixture (3) and the excipient-free spray dried BSAApi nanoparticles (4).

B) DSC spectra of apigenin (1), BSA (2), physical mixture (3) and the spray-dried BSA-Api nanoparticles with Lactohale $\mathbb{}(4)$.

C) DSC spectra of apigenin (1), BSA (2), physical mixture (3) and the spray-dried BSA-Api nanoparticles with L-leucine (4).

$42 \times 34 \mathrm{~mm}(600 \times 600 \mathrm{DPI})$ 


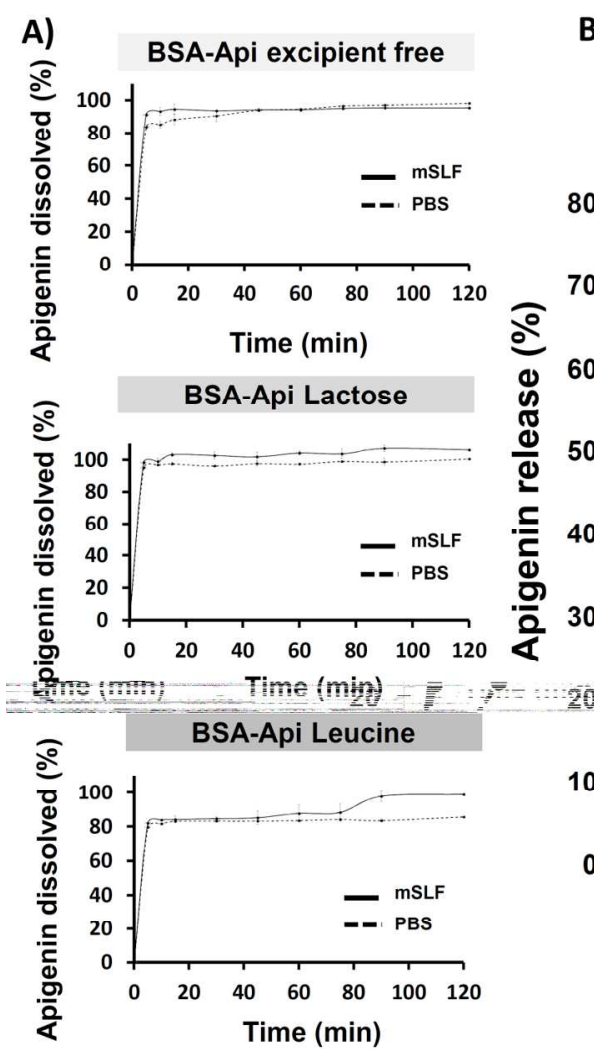

B)

80

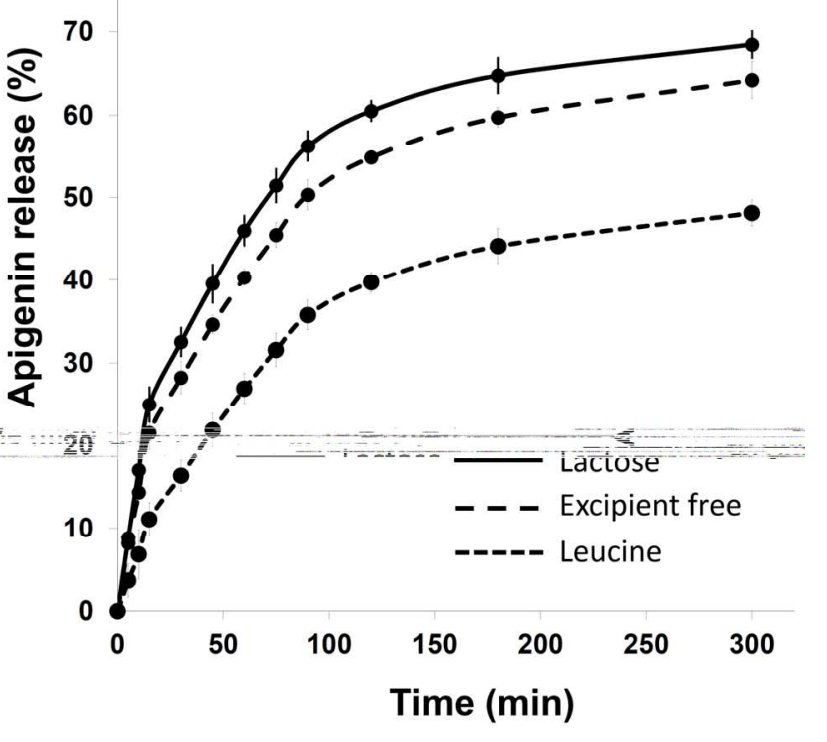

A) Solubility of spray dried BSA-Api formulations in PBS buffer and modified simulated lung fluid (mSLF).

B) Dissolution of apigenin from the formulations as a function of time in modified simulated lung fluid (mSLF).

$160 \times 128 \mathrm{~mm}(300 \times 300 \mathrm{DPI})$ 


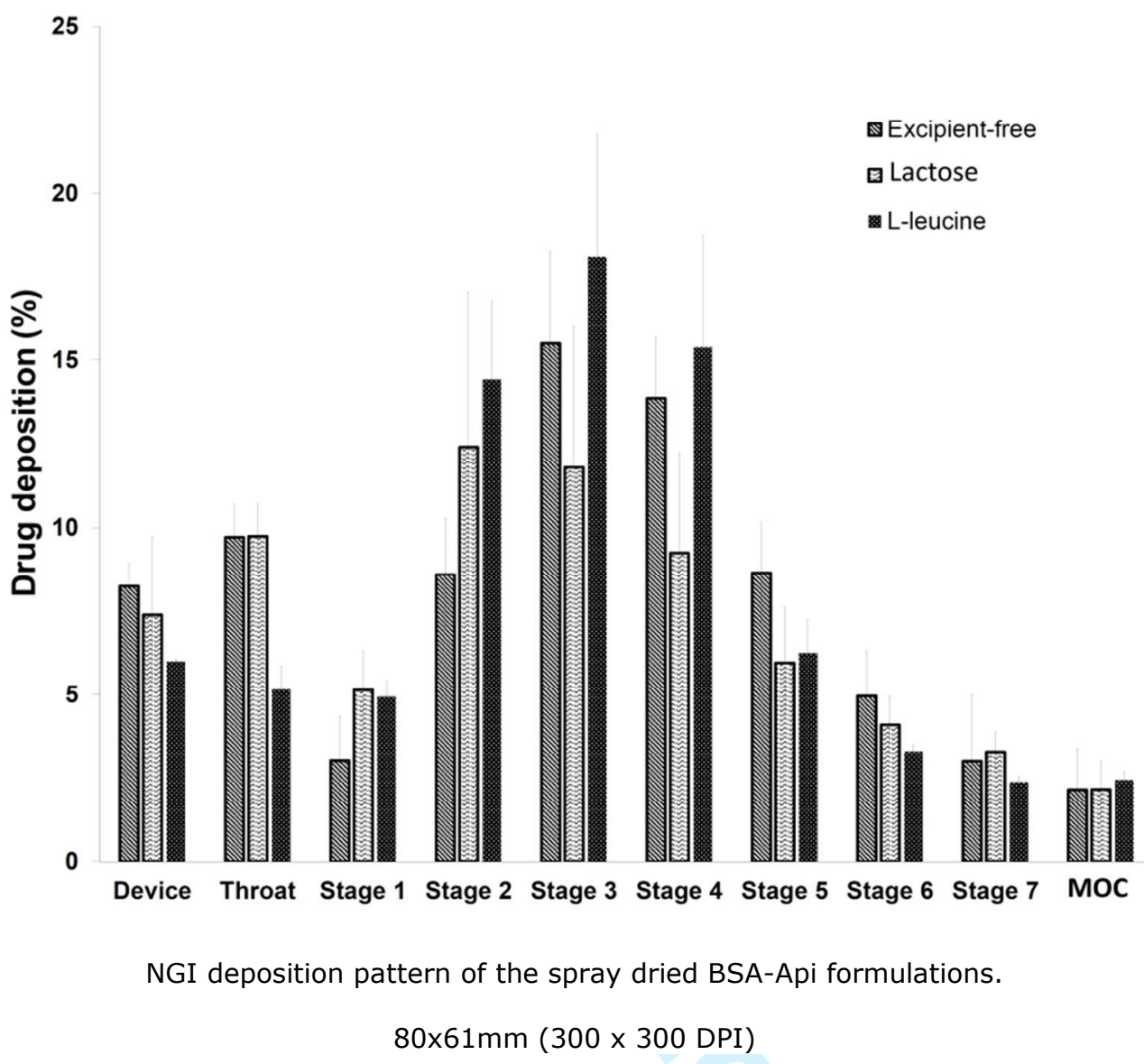



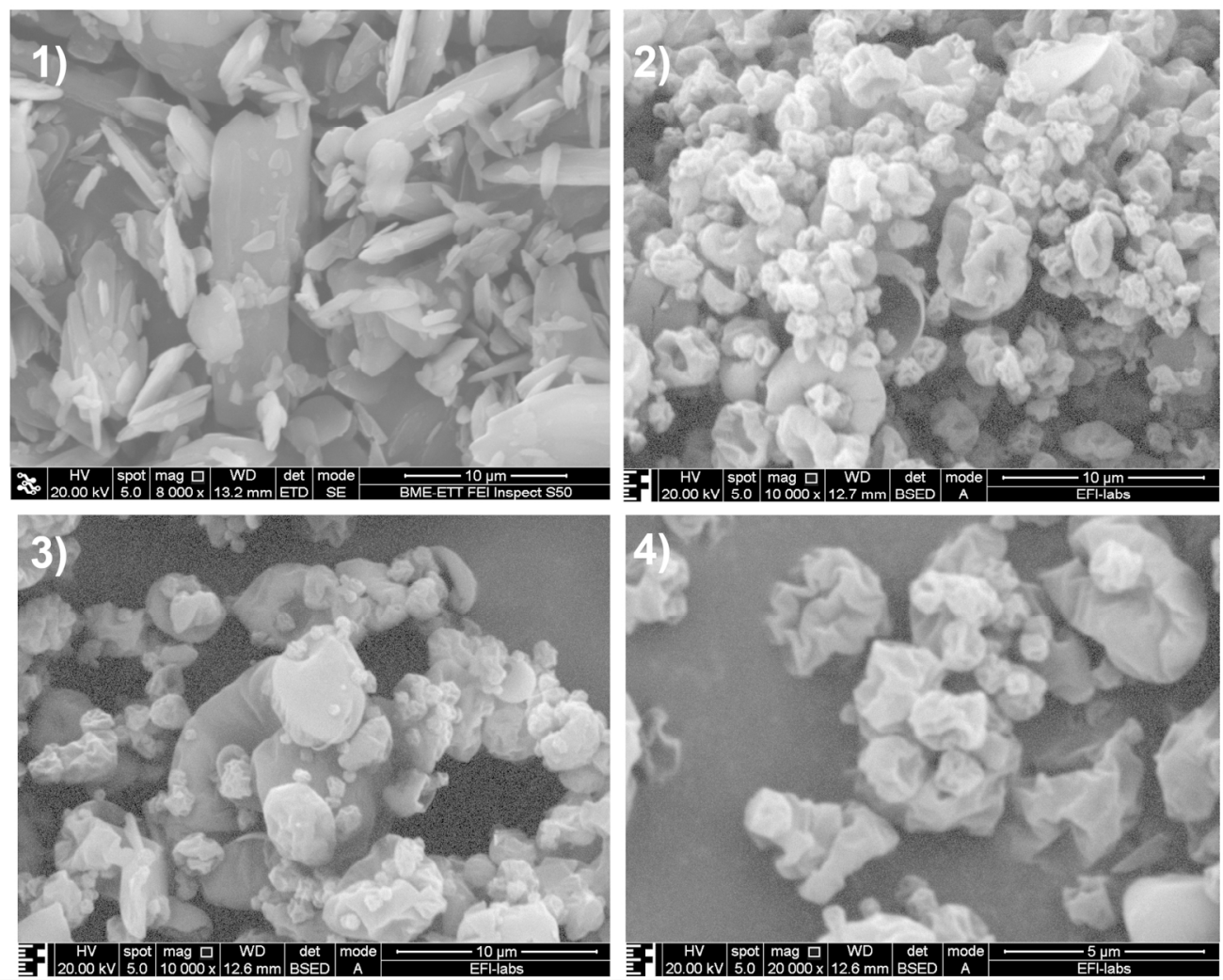

SEM images of raw apigenin (1), excipient-free spray dried BSA-Api nanoparticles (2), spray-dried BSA-Api nanoparticles with lactose (3), spray-dried BSA-Api nanoparticles with L-leucine (4) $20000 \times$ magnification.

$160 \times 127 \mathrm{~mm}(300 \times 300 \mathrm{DPI})$ 


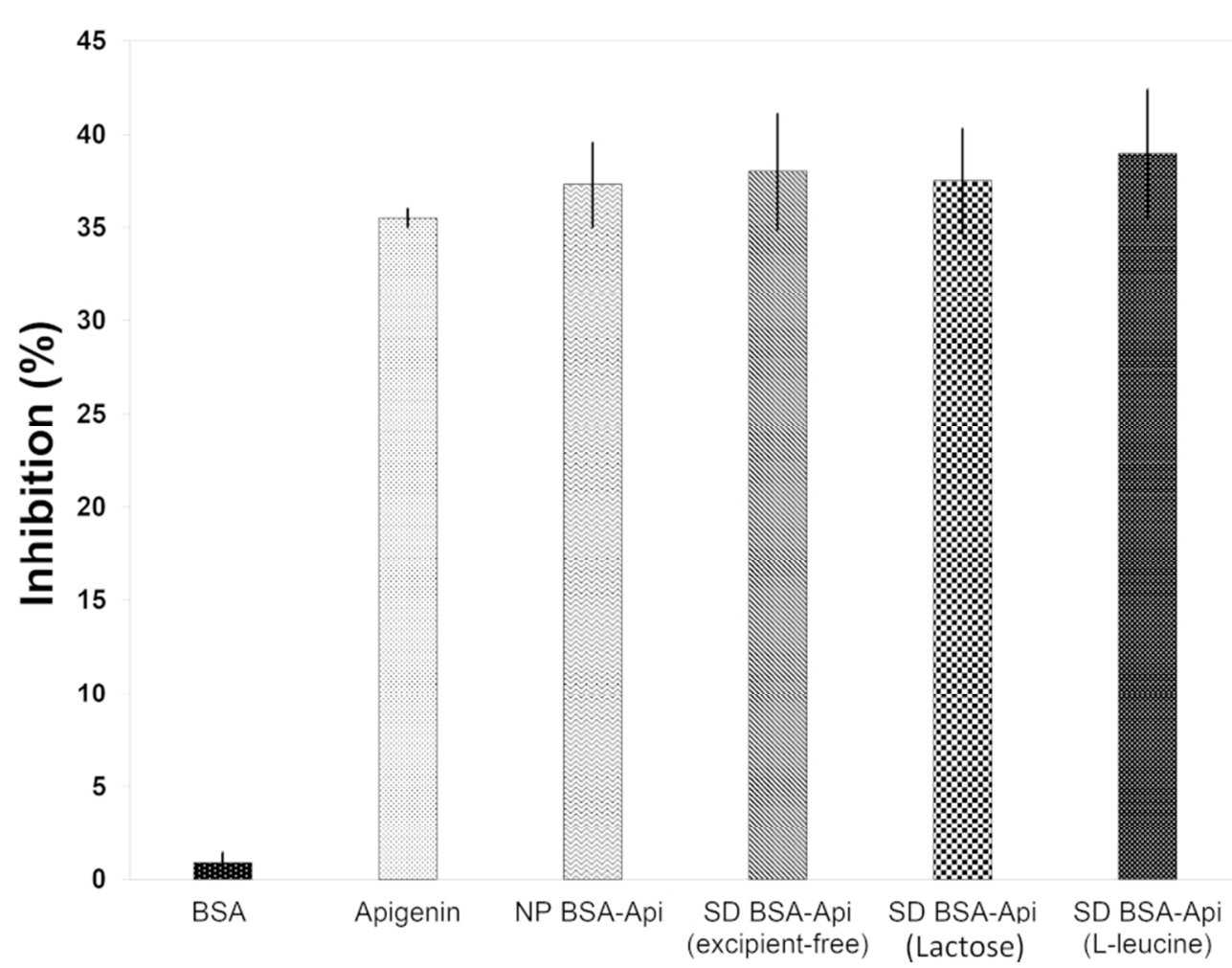

Radical scavenging activity of Apigenin solution, empty BSA nanoparticles, BSA-Apigenin nanoparticles (NP) and spray-dried nanoparticles (SD) with excipients. The antioxidant activity is expressed as the inhibition of $\mathrm{DPPH}^{*}$ free radical in percent.

$80 \times 60 \mathrm{~mm}(300 \times 300 \mathrm{DPI})$ 